\title{
Morse Index Theorem of Lagrangian Systems and Stability of Brake Orbit
}

\author{
Xijun $\mathrm{Hu}^{*} \quad \mathrm{Li} \mathrm{Wu}^{\dagger}$ Ran Yang ${ }^{\ddagger}$ \\ Department of Mathematics, Shandong University \\ Jinan, Shandong 250100, The People's Republic of China
}

\begin{abstract}
In this paper, we prove Morse index theorem of Lagrangian system with any self-adjoint boundary conditions. Based on it, we give some nontrivial estimation on the difference of Morse indices. As an application, we get a new criterion for the stability problem of brake periodic orbit.
\end{abstract}

AMS Subject Classification: 37B30, 53D12, 34B24, 37J25

Key Words. Morse index, Maslov index, Lagrangian systems, self-adjoint boundary conditions, stability of brake orbits

\section{Introduction}

In this paper, we consider the Morse index theorem of a Lagrangian system with general boundary conditions. Precisely, let

$$
\mathcal{F}(x)=\int_{0}^{T} L(t, x, \dot{x}) d t,
$$

where $x \in W^{1,2}\left([0, T], \mathbf{R}^{n}\right)$ and $L \in C^{2}\left([0, T] \oplus \mathbf{R}^{2 n}, \mathbf{R}\right)$ satisfying the Legendre convexity condition:

$$
\left(D_{v v}^{2} L(t, u, v) w, w\right)>0 \quad \text { for } \quad t \in[0, T], w \in \mathbf{R}^{n} \backslash\{0\},(u, v) \in \mathbf{R}^{n} \oplus \mathbf{R}^{n} .
$$

A solution $x$ of the corresponding Euler-Lagrange equation

$$
\frac{d}{d t} \frac{\partial L}{\partial \dot{x}}(t, x, \dot{x})-\frac{\partial L}{\partial x}(t, x, \dot{x})=0
$$

will be called a stationary point. Linearization of (1.2) along its stationary point is given by the following Sturm-Liouville system

$$
\mathcal{A} x(t):=-\frac{d}{d t}(P(t) \dot{x}(t)+Q(t) x(t))+Q(t)^{T} \dot{x}(t)+R(t) x(t)=0,
$$

*Partially supported by NSFC(No.11425105, 11790271), E-mail:xjhu@ sdu.edu.cn

†E-mail: 201790000005@sdu.edu.cn

E-mail: yangran201311260@ mail.sdu.edu.cn 
where $P(t)=\frac{\partial^{2} L}{\partial \dot{x}^{2}}, Q(t)=\frac{\partial^{2} L}{\partial x \partial \dot{x}}$ and $R(t)=\frac{\partial^{2} L}{\partial x^{2}}$. In fact, $\mathcal{A}$ can be considered as $\mathcal{F}^{\prime \prime}(x)$ which is the Hessian of $\mathcal{F}$ at $x$.

The boundary condition of (1.2) can be given in the following way. Let $\langle\cdot, \cdot\rangle$ be the standard Hermitian inner product in $\mathbf{C}^{2 n}$ and $J_{n}=\left[\begin{array}{cc}0 & -I_{n} \\ I_{n} & 0\end{array}\right]$, then $\left(\mathbf{C}^{2 n}, \omega\right)$ can be seen as a complex symplectic vector space with the symplectic form $\omega(x, y)=\langle J x, y\rangle$ for any $x, y \in \mathbf{C}^{2 n}$. A complex subspace $\Lambda$ is Lagrangian if and only if $\left.\omega\right|_{\Lambda}=0$ and $\operatorname{dim}_{\mathbf{C}} \Lambda=n$. We denote by $\operatorname{Lag}\left(\mathbf{C}^{2 n}, \omega\right)$ the set of Lagrangian subspaces. Let $y(t)=P(t) \dot{x}(t)+Q(t) x(t), z(t)=(y(t), x(t))^{T}$, then we consider the most general self-adjoint boundary conditions, namely,

$$
(z(0), z(T)) \in \Lambda_{0},
$$

where $\Lambda_{0} \in \operatorname{Lag}\left(\mathbf{C}^{2 n} \oplus \mathbf{C}^{2 n},-\omega \oplus \omega\right)$. Obviously, $\mathcal{A}$ is a self-adjoint operator on $L^{2}\left([0, T], \mathbf{C}^{2 n}\right)$ with domain

$$
\left.E_{\Lambda_{0}}(0, T):=\left\{x \in W^{2,2}\left([0, T], \mathbf{C}^{2 n}\right),(z(0), z(T)) \in \Lambda_{0}\right)\right\}
$$

For a self-adjoint operator $A$, we denote its Morse index by $m^{-}(A)$, which is the number of total negative eigenvalues of $A$. Throughout of the paper, we always denote $m^{0}(A)=\operatorname{dim} \operatorname{ker}(A)$ and $m^{+}(A)$ be the total number of positive eigenvalues of $A$. For a critical point $x$ of $\mathcal{F}$, we define the Morse index by

$$
m^{-}\left(x, \Lambda_{0}\right)=m^{-}(\mathcal{A})
$$

and we always omit $x$ when there is no confusion.

Obviously, the Morse index depends on the boundary condition $\Lambda_{0}$. Let $\Lambda_{N}^{n}=\{0\} \oplus \mathbf{C}^{n}, \Lambda_{D}^{n}=\mathbf{C}^{n} \oplus\{0\}$, which is Lagrangian subspace of $\left(\mathbf{C}^{2 n}, \omega\right)$ and can be considered as the Neumann and Dirichlet boundary conditions. For convenience, we set $\Lambda_{D}=\Lambda_{D}^{n} \oplus \Lambda_{D}^{n}$ and $\Lambda_{N}=\Lambda_{N}^{n} \oplus \Lambda_{N}^{n}$. Obviously,

$$
\Lambda_{N}=\left\{(z(0), z(T)) \in \mathbf{C}^{4 n}, y(0)=y(T)=0\right\}, \quad \Lambda_{D}=\left\{(z(0), z(T)) \in \mathbf{C}^{4 n}, x(0)=x(T)=0\right\},
$$

which means both start time and end time with Neumann or Dirichlet boundary conditions.

It is well known that the Morse index with Dirichlet boundary condition can be expressed as sum of conjugate points, please refer [13] and reference therein for the detail. More precisely, by the standard Legendre transformation, the system $(1.3)$ becomes into

$$
\dot{z}(t)=J \mathcal{B}(t) z(t)
$$

where

$$
\mathcal{B}(t)=\left[\begin{array}{cc}
P^{-1}(t) & -P^{-1}(t) Q(t) \\
-Q^{T}(t) P^{-1}(t) & Q^{T}(t) P^{-1}(t) Q(t)-R(t)
\end{array}\right] .
$$

Let $\gamma(t)$ be the fundamental solution of 1.5 , that is $\gamma(0)=I_{2 n}$ and $\dot{\gamma}(t)=J \mathcal{B}(t) \gamma(t)$. It is well known that

$$
\gamma(t) \in \operatorname{Sp}(2 n):=\left\{M^{T} J M=J, M \in G L\left(\mathbf{R}^{2 n}\right)\right\} .
$$

We have the well known Morse index Theorem

$$
m^{-}\left(\Lambda_{D}\right)=\sum_{0<t_{0}<T} \operatorname{dim} \operatorname{ker} \gamma\left(t_{0}\right) \Lambda_{D} \cap \Lambda_{D}
$$

For general boundary condition $\Lambda_{0}$, we can't get a simple formula as in 1.7). 
In order to give the Morse index Theorem with general boundary conditions, we consider the difference of $m^{-}\left(\Lambda_{0}\right)-m^{-}\left(\Lambda_{D}\right)$. The difference is expressed by Duistermaat triple index [9]. Let $\alpha, \beta$ and $\delta$ be three isotropic subspaces of complex symplectic vector space $(V, \omega)$, the triple index $i(\alpha, \beta, \delta)$ is well defined [25] and satisfied

$$
0 \leq i(\alpha, \beta, \delta) \leq \operatorname{dim} \alpha-\operatorname{dim}(\alpha \cap \beta+\beta \cap \delta) .
$$

For a matrix $M \in L\left(\mathbf{C}^{m}\right)$, we always denote by

$$
G r(M):=\left\{(x, M x), x \in \mathbf{C}^{m}\right\},
$$

which is a linear subspace of $\mathbf{C}^{m} \oplus \mathbf{C}^{m}$. Since $\gamma(T) \in \mathrm{Sp}(2 n)$, it is obvious that $\operatorname{Gr}(\gamma(T)) \in \operatorname{Lag}\left(\mathbf{C}^{2 n} \oplus\right.$ $\left.\mathbf{C}^{2 n},-\omega \oplus \omega\right)$, and $i\left(\operatorname{Gr}(\gamma(T)), \Lambda_{0}, \Lambda_{D}\right)$ is well defined.

Theorem 1.1. For a critical point $x$ of the Lagrangian system (1.1), the Morse indices $m^{-}\left(\Lambda_{0}\right)$ and $m^{-}\left(\Lambda_{D}\right)$ satisfy

$$
m^{-}\left(\Lambda_{0}\right)-m^{-}\left(\Lambda_{D}\right)=i\left(\operatorname{Gr}(\gamma(T)), \Lambda_{0}, \Lambda_{D}\right)
$$

Let $V\left(\Lambda_{0}\right)$ be the subspace of $\Lambda_{N}$ defined by

$$
V\left(\Lambda_{0}\right)=\left(\Lambda_{0}+\Lambda_{D}\right) \cap \Lambda_{N}
$$

then $(x(0), x(T))^{T} \in V\left(\Lambda_{0}\right)$. We always denote

$$
v\left(\Lambda_{0}\right)=\operatorname{dim} V\left(\Lambda_{0}\right)
$$

Obviously, $v\left(\Lambda_{D}\right)=0$ and $v\left(\Lambda_{N}\right)=2 n$. Direct computations show that for the periodic boundary condition $\Lambda_{P}, v\left(\Lambda_{P}\right)=n$.

Please note that (??) implies

$$
i\left(G r(\gamma(T)), \Lambda_{0}, \Lambda_{D}\right) \leq v\left(\Lambda_{0}\right),
$$

we have the following inequality.

\section{Corollary 1.2.}

$$
m^{-}\left(\Lambda_{D}\right) \leq m^{-}\left(\Lambda_{0}\right) \leq m^{-}\left(\Lambda_{D}\right)+v\left(\Lambda_{0}\right) .
$$

It is well known that for Dirichlet boundary condition the Morse index has some monotone property [8]. To be precisely, for the linear system (1.3) defined on time interval $[a, b]$. Let

$$
m_{[a, b]}^{-}\left(\Lambda_{0}\right)=m^{-}\left(\left.\mathcal{A}\right|_{E_{\Lambda_{0}}(a, b)}\right) \text {. }
$$

For $[c, d] \subset[a, b]$, we have

$$
m_{[a, b]}^{-}\left(\Lambda_{D}\right) \geq m_{[a, c]}^{-}\left(\Lambda_{D}\right)+m_{[c, d]}^{-}\left(\Lambda_{D}\right)+m_{[d, b]}^{-}\left(\Lambda_{D}\right) .
$$

This property is not true for general boundary conditions, instead, as a corollary of (1.11), we have the next estimation.

\section{Corollary 1.3.}

$$
m_{[a, b]}^{-}\left(\Lambda_{0}\right) \geq m_{[c, d]}^{-}\left(\Lambda_{0}\right)+m_{[a, c]}^{-}\left(\Lambda_{D}\right)+m_{[d, b]}^{-}\left(\Lambda_{D}\right)-v\left(\Lambda_{0}\right)
$$


Let $\Lambda_{s} \in \operatorname{Lag}\left(\mathbf{C}^{n},-\omega\right)$ and $\Lambda_{e} \in \operatorname{Lag}\left(\mathbf{C}^{n}, \omega\right)$, then the separated boundary conditions can be given by $\Lambda_{0}=\Lambda_{s} \oplus \Lambda_{e}$. The $\Lambda_{s}$ conjugate point is defined by $\gamma(t) \Lambda_{s} \cap \Lambda_{D}^{n} \neq\{0\}$. We give the general Morse index Theorem as follows

Theorem 1.4. For a critical point $x$ of the Lagrangian system (1.1) with the separated boundary condition, the Morse index $m^{-}\left(\Lambda_{s} \oplus \Lambda_{e}\right)$ satisfies

$$
m^{-}\left(\Lambda_{s} \oplus \Lambda_{e}\right)=\sum_{0<t_{0}<T} \operatorname{dim}\left(\gamma\left(t_{0}\right) \Lambda_{s} \cap \Lambda_{D}^{n}\right)+i\left(\gamma(T) \Lambda_{s}, \Lambda_{e}, \Lambda_{D}^{n}\right) .
$$

Remark 1.5. Theorem 1.4 can be used to study the Morse index of geodesics on Riemannian manifold. The classical Morse index theorem for a Riemannian manifold $(M, g)$ can be traced back to [19]. The generalizations of this result are referred to [2], [4], [10], [14], [23] and reference therein. Kalish [14] proved the Morse index theorem where the end points are in submanifolds. As a corollary of Theorem 1.4 we generalize Kalish's results to the degenerate case. Please refer to Example 3.8 for the details.

The Morse index under the general boundary condition can be expressed by the Maslov index. This is first studied by Duistermaat [9]. In the periodic boundary condition it is proved by [15], [16], [1]. In [13], $\mathrm{Hu}$ and Sun give a clear form for the case of the boundary condition given by $(x(0), x(T)) \in V \subset \Lambda_{N}$. More related results can be found in [26] and the reference therein.

For a solution $z$ of $(1.5]$, its Maslov index (refer to [3], [7], [13]) is given by

$$
\mu^{-}(z)=\mu\left(\Lambda_{0}, G r(\gamma(t)), t \in[0, T]\right) .
$$

As a corollary of Theorem 1.1 we can get the relationship between the Maslov index and Morse index for the boundary condition (1.4).

Corollary 1.6. For a critical point $x$ of the Lagrangian system (1.1) with the boundary condition (1.4), its Morse index $\mathrm{m}^{-}(x)$ and Maslov index $\mu^{-}(z)$ satisfy

$$
\mu^{-}(z)-m^{-}(x)=n-i\left(\operatorname{Gr}\left(I_{2 n}\right), \Lambda_{0}, \Lambda_{D}\right)
$$

Since $0 \leq i\left(G r\left(I_{2 n}\right), \Lambda_{0}, \Lambda_{D}\right) \leq 2 n$ for any $\Lambda_{0}$, we have

$$
-n \leq \mu^{-}(z)-m^{-}(x) \leq n .
$$

Example 1.7. Denote the difference between the Maslov index and Morse index by $\Delta$. By Corollary 1.6 we list several common examples to compute associated $\Delta$.

1. [13, Theorem1.2] Let $V$ be any subspace of $\Lambda_{N}$ and the boundary condition is given by $\Lambda_{V}=$ $\mathcal{J} V^{\perp} \oplus V$, where $\mathcal{J}=-J \oplus J$. Then

$$
i\left(G r\left(I_{2 n}\right), \Lambda_{V}, \Lambda_{D}\right)=n-\operatorname{dim}\left(V^{\perp} \cap G r\left(-I_{n}\right)\right), \quad \Delta\left(\Lambda_{V}\right)=\operatorname{dim}\left(V^{\perp} \cap G r\left(-I_{n}\right)\right) .
$$

Follows [13], we consider two important cases. The first case is given by $V=G r(M)$, where $M \in G L\left(\mathbf{C}^{n}\right)$. For this case, $\operatorname{dim}\left(V^{\perp} \cap \operatorname{Gr}\left(-I_{n}\right)\right)=\operatorname{dim}\left(M-I_{n}\right)$. Then

$$
\Delta\left(\Lambda_{V}\right)=\operatorname{dim}\left(M-I_{n}\right) .
$$


The second case is given by $V=V_{1} \oplus V_{2}$, where $V_{1}, V_{2}$ be two subspaces of $\mathbf{C}^{n}$. For this case $\operatorname{dim}\left(V^{\perp} \cap\right.$ $\left.\operatorname{Gr}\left(-I_{n}\right)\right)=\operatorname{dim}\left(V_{1}^{\perp} \cap V_{2}^{\perp}\right)$. Then

$$
\Delta\left(\Lambda_{V}\right)=\operatorname{dim}\left(V_{1}^{\perp} \cap V_{2}^{\perp}\right)
$$

As special cases, we have

Dirichlet boundarycondition $i\left(G r\left(I_{2 n}\right), \Lambda_{D}, \Lambda_{D}\right)=0, \quad \Delta\left(\Lambda_{D}\right)=n$.

Neumann boundarycondition $i\left(\operatorname{Gr}\left(I_{2 n}\right), \Lambda_{N}, \Lambda_{D}\right)=n, \quad \Delta\left(\Lambda_{N}\right)=0$.

Periodic boundarycondition $i\left(G r\left(I_{2 n}\right), \Lambda_{P}, \Lambda_{D}\right)=0, \quad \Delta\left(\Lambda_{P}\right)=n$.

2. Separated boundary condition

For this case, we have

$$
i\left(G r\left(I_{2 n}\right), \Lambda_{s} \oplus \Lambda_{e}, \Lambda_{D}\right)=n-\operatorname{dim}\left(\Lambda_{s} \cap \Lambda_{D}^{n}\right)+i\left(\Lambda_{s}, \Lambda_{e}, \Lambda_{D}^{n}\right)
$$

and

$$
\Delta\left(\Lambda_{s} \oplus \Lambda_{e}\right)=\operatorname{dim}\left(\Lambda_{s} \cap \Lambda_{D}^{n}\right)-i\left(\Lambda_{s}, \Lambda_{e}, \Lambda_{D}^{n}\right) .
$$

Particularly, for the special case where $y(0)=A_{s} x(0), y(T)=A_{e} x(T)$, we have

$$
i\left(G r\left(I_{2 n}\right), \Lambda_{s} \oplus \Lambda_{e}, \Lambda_{D}\right)=n+m^{+}\left(A_{e}-A_{s}\right), \quad \Delta\left(\Lambda_{s} \oplus \Lambda_{e}\right)=-m^{+}\left(A_{e}-A_{s}\right) .
$$

Note that for this special kind of separated boundary condition, we always have $\mu^{-}(z) \leq m^{-}\left(\Lambda_{s} \oplus \Lambda_{e}\right)$. Moreover, if we take $A_{s}=0, A_{e}=I_{n}$, then $\mu^{-}(z)-m^{-}\left(\Lambda_{s} \oplus \Lambda_{e}\right)=-n$.

Recently, the authors studied the relationship between Maslov index and Morse index for Schrödinger operators on finite interval $[0,1]$ in [11] under the separated boundary condition. In above example, we give a different proof by using our general result.

As an application, we consider the stability problem of brake periodic orbits. For a periodic orbit $x$ with fundamental solution $\gamma(t), x$ is called spectral stable if $\sigma(\gamma(T)) \subset \mathbf{U}$, is called linear stable if moreover $\gamma(T)$ is semi-simple.

For a brake periodic orbit, $P(t), Q(t)$ and $R(t)$ are all real $T$-periodic symmetric matrices and satisfy the following condition:

$$
P(-t)=P(t), \quad Q(-t)=-Q(t), \quad R(-t)=R(t), \quad \forall t \in \mathbf{R} .
$$

As an application of Theorem 1.1, we can give an estimation of the geometrical multiplicity of all non-real eigenvalues of $\gamma(T)$.

Theorem 1.8. Let $\mathbf{C}^{\perp}=\{\lambda \in \mathbf{C} \mid \operatorname{Im}(\lambda)>0\}$, for the brake orbit, we have

$$
\operatorname{dim} \bigoplus_{\lambda \in \sigma(\gamma(T)) \cap\left(\mathbf{C}^{\perp} \cup\{ \pm 1\}\right)} \operatorname{ker}\left(\gamma(T)-\lambda I_{2 n}\right) \leq m^{-}\left(\Lambda_{N}\right)+m^{0}\left(\Lambda_{N}\right)-m^{-}\left(\Lambda_{D}\right) .
$$

and

$$
\operatorname{dim} \bigoplus_{\lambda \in \sigma(\gamma(T)) \cap \mathbf{C}^{\perp}} \operatorname{ker}\left(\gamma(T)-\lambda I_{2 n}\right) \leq m^{-}\left(\Lambda_{N}\right)-m^{-}\left(\Lambda_{D}\right) .
$$

Please note that, if $m^{-}\left(\Lambda_{N}\right)=m^{-}\left(\Lambda_{D}\right)$, then $\sigma(\gamma(T)) \subset \mathbf{R}$. As a corollary of Theorem 1.8, we have the next estimation. 
Theorem 1.9. For a given brake orbit x of system (1.3), we assume $m^{-}\left(x, \Lambda_{P}\right)=k$, then for the monodromy matrix $\gamma(T)$ we have

$$
\operatorname{dim} \bigoplus_{\lambda \in \sigma(\gamma(T)) \cap \mathbf{C}^{\perp}} \operatorname{ker}\left(\gamma(T)-\lambda I_{2 n}\right) \leq 2 k .
$$

In 1987, Offin proved the non-degenerate brake orbit with zero Morse index is hyperbolic [20], some related results can be found in [6]. Recently, Ureña proved [22, Theorem 1.1] that all the eigenvalues of $\gamma(T)$ are not only real but also positive. Inequality (1.24) can be considered as a generalization of their results. In the case $m^{-}\left(x, \Lambda_{P}\right)=0$, we can get Ureña's result by combining with other simple discussions, the details will be given in Section 4 .

This paper is organized as follows, in Section 2, we briefly review the index theory for Lagrangian subspace, and we prove the Morse index theorem in Section 3, at last,we prove the stability theorem of brake orbits.

\section{Index Theory for the Lagrangian subspaces}

In this section, we briefly review the theory about Maslov index, Hörmander index, triple index and their relations.

In Hamiltonian system theory, Maslov index is an important topological characterization. About this theory, readers are referred to [3], [9], [21] and so on. Denote $\Lambda^{ \pm}=\operatorname{ker}\left(i J \mp I_{2 n}\right)$, then $\Lambda$ is Lagrangian if and only if $\Lambda$ can be expressed as a graph of a unitary operator $U: \Lambda^{+} \rightarrow \Lambda^{-}$. So we can define a homeomorphic (isomorphic) map $f: \operatorname{Lag}\left(\mathbf{C}^{2 n}, \omega\right) \rightarrow \mathbf{U}(n)$. In fact, $f$ can be defined in the following way. Denote the conjugate transpose of a complex matrix $X$ by $X^{*}$. Recall that the Lagrangian frame of a given Lagrangian subspace $\Lambda$ is an injective linear map $\mathcal{Z}: \mathbf{C}^{n} \rightarrow \Lambda$ with the form $\mathcal{Z}=\left[\begin{array}{l}X \\ Y\end{array}\right]$, where $X, Y$ are $n \times n$ complex matrices such that $X^{*} Y=Y^{*} X$ and $\operatorname{rank}(\mathcal{Z})=n$. To be convenient, we will denote Lagrangian subspace only by its Lagrangian frame later.

$$
f(\mathcal{Z})=(X-i Y)(X+i Y)^{-1} .
$$

Note that

$$
\operatorname{dim}\left(\Lambda_{1} \cap \Lambda_{2}\right)=\operatorname{dim} \operatorname{ker}\left(f\left(\Lambda_{2}\right)^{-1} f\left(\Lambda_{1}\right)-I_{n}\right) .
$$

Then for any fixed $U_{0} \in \mathbf{U}(n)$, we can define the singular cycle $\Sigma_{U_{0}}$ of $U_{0}$ as

$$
\Sigma_{U_{0}}=\left\{U \in \mathbf{U}(n) \mid \operatorname{det}\left(U_{0}^{-1} U-I_{n}\right)=0\right\} .
$$

Let $U_{t}(t \in[a, b])$ be any path in $\mathbf{U}(n)$. By the proper small perturbation $e^{i s} U_{t}(|s| \leq \varepsilon)$, for any fixed $t_{0} \in[a, b]$, we have the path $e^{i s} U_{t_{0}}$ is transversal to $\Sigma_{U_{0}}$ and for fixed small enough $s_{0}$, there holds $e^{-i s_{0}} U_{a}$ and $e^{-i s_{0}} U_{b}$ are all not in the singular cycle of $U_{0}$. So the intersection number $\left[e^{-i s_{0}} U_{t}: \Sigma_{U_{0}}\right]$ is well defined. Then we can introduce the following definition:

Definition 2.1. Let $\Lambda(t)$ be a path in $\operatorname{Lag}\left(\mathbf{C}^{2 n}\right)$ and $\Lambda_{0} \in \operatorname{Lag}\left(\mathbf{C}^{2 n}\right)$, then the Maslov index is defined as

$$
\mu\left(\Lambda_{0}, \Lambda(t)\right):=\left[e^{-i s_{0}} f(\Lambda(t)): \Sigma_{f\left(\Lambda_{0}\right)}\right]
$$


[21] gives an effective way to compute the Maslov index $\mu\left(\Lambda_{0}, \Lambda(t)\right)$ by using crossing form. For the $C^{1}$-Lagrangian path $\{\Lambda(t), t \in[0, T]\}$, then $t_{0}$ is called a crossing if $\Lambda\left(t_{0}\right) \cap \Lambda_{0} \neq 0$. Let $v$ be any vector in $\Lambda\left(t_{0}\right) \cap \Lambda_{0}$ and $V_{t_{0}}$ be a fixed Lagrangian subspace which is transversal to $\Lambda\left(t_{0}\right)$. For small $t$, the crossing form is defined by

$$
\Gamma\left(\Lambda\left(t_{0}\right), \Lambda_{0}, t_{0}\right)(v)=\left.\frac{d}{d t}\right|_{t=t_{0}} \omega(v, u(t))
$$

where $u(t) \in V_{t_{0}}$ such that $v+u(t) \in \Lambda(t)$ and the form is independent of the choice of $V_{t_{0}}$. For the special Lagrangian path $\Lambda(t)=\gamma(t) W$, where $\gamma(t) \in \mathrm{Sp}(2 n)$ and $W$ is a fixed Lagrangian subspace, then the crossing form is $\left\langle-\gamma(t)^{T} J \dot{\gamma}(t) v, v\right\rangle$ for $v \in \gamma(t)^{-1}(\Lambda(t) \cap W)$.

A crossing is called regular if the crossing form is non-degenerate. For every $C^{1}$ path with fixed endpoints, we can make sure that all the crossings are regular by small perturbation. Then following [17] we have

$$
\mu\left(\Lambda_{0}, \Lambda(t)\right)=m^{+}\left(\Gamma\left(\Lambda(0), \Lambda_{0}, 0\right)\right)+\sum_{t \in \mathcal{S}} \operatorname{sgn}\left(\Gamma\left(\Lambda(t), \Lambda_{0}, t\right)\right)-m^{-}\left(\Gamma\left(\Lambda(T), \Lambda_{0}, T\right)\right),
$$

where $\mathcal{S}$ is the set of all crossings and $m^{+}, m^{-}$are the dimensions of positive and negative subspaces respectively.

There is another important index related to the Maslov index, namely, the Hörmander index (See [21]). Let $V_{0}, V_{1}, \Lambda_{0}, \Lambda_{1}$ be four Lagrangian subspaces and $\{\Lambda(t), t \in[0, T]\}$ is any Lagrangian path such that $\Lambda_{0}=\Lambda(0), \Lambda_{1}=\Lambda(T)$, then there is the following definition:

Definition 2.2. Let $V_{0}, V_{1}, \Lambda_{0}, \Lambda_{1}$ and $\Lambda(t)$ be as above, the Hörmander index is defined to be

$$
s\left(\Lambda_{0}, \Lambda_{1} ; V_{0}, V_{1}\right):=\mu\left(V_{1}, \Lambda(t)\right)-\mu\left(V_{0}, \Lambda(t)\right) .
$$

Please note that the symbol $s\left(\Lambda_{0}, \Lambda_{1} ; V_{0}, V_{1}\right)$ here is a little different from the original one in [21] in order to correspond to [25, Definition 3.9]. One can check that the above definition is independent on the choice of path $\Lambda(t)$, so it's well defined. Recently, in [25], the second author and his collaborators studied the Hörmander index in the finite dimensional case by using triple index.

Now we will introduce the definition of triple index. Generally, let $\alpha, \beta$ and $\delta$ be three isotropic subspaces of complex symplectic vector space $(V, \omega)$, then define the form $\mathfrak{Q}:=\mathfrak{Q}(\alpha, \beta ; \delta)$ on $\alpha \cap(\beta+\delta)$ by

$$
\mathfrak{Q}\left(x_{1}, x_{2}\right)=\omega\left(y_{1}, z_{2}\right),
$$

where $x_{j}=y_{j}+z_{j} \in \alpha \cap(\beta+\delta)$ and $y_{j} \in \beta, z_{j} \in \delta$ for $j=1$,2. From [25], when $\alpha, \beta, \delta$ are Lagrangian subspaces, we have

$$
\operatorname{ker} \mathfrak{Q}(\alpha, \beta, \delta)=\alpha \cap \beta+\alpha \cap \delta .
$$

Moreover, we need the following lemma [25, Lemma 3.2]:

Lemma 2.3. For three isotropic subspaces $\alpha, \beta, \delta$, let $\mathfrak{Q}_{1}:=\mathbb{Q}(\alpha, \beta ; \delta), \mathfrak{Q}_{2}:=\mathbb{Q}(\beta, \delta ; \alpha)$ and $\mathfrak{Q}_{3}:=$ $\mathfrak{Q}(\delta, \alpha ; \beta)$, then we have

$$
m^{ \pm}\left(\mathfrak{Q}_{1}\right)=m^{ \pm}\left(\mathfrak{Q}_{2}\right)=m^{ \pm}\left(\mathfrak{Q}_{3}\right)
$$

Now by equation (2.6) of [9], the triple index is well-defined as following: 
Definition 2.4. Let $\alpha, \beta$ and $\kappa$ be three Lagrangian subspaces of complex symplectic vector space $(V, \omega)$, then the triple index of $\alpha, \beta, \kappa$ is defined by

$$
i(\alpha, \beta, \kappa)=m^{-}(\mathfrak{Q}(\alpha, \delta ; \beta))+m^{-}(\mathfrak{Q}(\beta, \delta ; \kappa))-m^{-}(\mathfrak{Q}(\alpha, \delta ; \kappa)),
$$

where $\delta$ is a Lagrangian subspace such that $\delta \cap \alpha=\delta \cap \beta=\delta \cap \kappa=\{0\}$.

An equivalent definition can be given as follows

$$
\left.i(\alpha, \beta, \kappa)=m^{+}(\mathfrak{Q}(\alpha, \beta ; \kappa))+\operatorname{dim}(\alpha \cap \kappa)-\operatorname{dim}(\alpha \cap \beta \cap \kappa)\right),
$$

where $\mathrm{m}^{+}$denotes the dimension of maximal positive definite subspace which $\mathfrak{Q}$ acts on. It follows that

$$
i(\alpha, \beta, \kappa) \geq 0 .
$$

The triple index $i(\alpha, \beta, \kappa)$ can be calculated and estimated by [25, Lemma 3.13]:

$$
\begin{aligned}
i(\alpha, \beta, \kappa) & \left.=m^{+}(\mathfrak{Q}(\alpha, \beta ; \kappa))+\operatorname{dim}(\alpha \cap \kappa)-\operatorname{dim}(\alpha \cap \beta \cap \kappa)\right) \\
& \leq \operatorname{dim} \alpha-\operatorname{dim}(\alpha \cap \beta)-\operatorname{dim}(\beta \cap \kappa)+\operatorname{dim}(\alpha \cap \beta \cap \kappa) \\
& =\operatorname{dim} \alpha-\operatorname{dim}(\alpha \cap \beta+\beta \cap \kappa),
\end{aligned}
$$

In particular, we have [25, Corollary 3.14]

$$
i(\alpha, \alpha, \beta)=i(\beta, \alpha, \alpha)=0, \quad i(\alpha, \beta, \alpha)=\operatorname{dim} \alpha-\operatorname{dim}(\alpha \cap \beta) .
$$

For four given Lagrangian subspaces $\lambda_{1}, \lambda_{2}, \kappa_{1}, \kappa_{2}$ of complex symplectic vector space $(V, \omega)$, the main theorem 1.1 of [25] gives an efficient way to compute the Hörmander index $s\left(\lambda_{1}, \lambda_{2} ; \kappa_{1}, \kappa_{2}\right)$ by

$$
s\left(\lambda_{1}, \lambda_{2} ; \kappa_{1}, \kappa_{2}\right)=i\left(\lambda_{1}, \lambda_{2}, \kappa_{2}\right)-i\left(\lambda_{1}, \lambda_{2}, \kappa_{1}\right)=i\left(\lambda_{1}, \kappa_{1}, \kappa_{2}\right)-i\left(\lambda_{2}, \kappa_{1}, \kappa_{2}\right) .
$$

In fact, the Maslov index can be expressed by the triple index.

Lemma 2.5. For $\Lambda, \Lambda_{0}, V(t), t \in[a, b]$ are Lagrangian subspaces. If $V(t)$ transversal to $\Lambda$ for $t \in[a, b]$, then

$$
\mu\left(\Lambda_{0}, V(t) ;[a, b]\right)=i\left(V(b), \Lambda_{0}, \Lambda\right)-i\left(V(b), \Lambda_{0}, \Lambda\right) .
$$

Proof. By easy computation

$$
\begin{aligned}
\mu\left(\Lambda_{0}, V(t) ;[a, b]\right) & =\mu\left(\Lambda_{0}, V(t) ;[a, b]\right)-\mu(\Lambda, V(t) ;[a, b]) \\
& =s\left(V(a), V(b) ; \Lambda, \Lambda_{0}\right) \\
& =i\left(V(a), \Lambda, \Lambda_{0}\right)-i\left(V(b), \Lambda, \Lambda_{0}\right) .
\end{aligned}
$$

In order to compute $i\left(G r(\gamma(T)), \Lambda_{0}, \Lambda_{D}\right)$, we firstly change the basis of $\left(\mathbf{C}^{2 n} \oplus \mathbf{C}^{2 n},-\omega \oplus \omega\right)$ such that the symplectic structure with the standard form $J_{2 n}$. In fact, let $S=\left[\begin{array}{cccc}-I_{n} & 0 & 0 & 0 \\ 0 & 0 & I_{n} & 0 \\ 0 & I_{n} & 0 & 0 \\ 0 & 0 & 0 & I_{n}\end{array}\right]$, then

$$
S^{T} \mathcal{J} S=J_{2 n}
$$


$\Lambda_{0}$ can be expressed more explicitly. Note that the boundary condition (1.4) is equivalent to

$$
(-y(0), y(T), x(0), x(T))^{T} \in \Lambda_{0}
$$

under the new basis. According to the splitting $\mathbf{C}^{2 n} \cong \Lambda_{N}=V \oplus V^{\perp}$, we can split $(-y(0), y(T))^{T}=$ $\left(-y_{1}(0), y_{1}(T)\right)^{T}+\left(-y_{2}(0), y_{2}(T)\right)^{T}$, where $\left(-y_{1}(0), y_{1}(T)\right)^{T} \in J_{2 n} V^{\perp}$ and $\left(-y_{2}(0), y_{2}(T)\right)^{T} \in J_{2 n} V$, then we have a matrix $A$ from $V$ to $J_{2 n} V$ such that $\left(-y_{2}(0), y_{2}(T)\right)^{T}=A(x(0), x(T))^{T}$. Since $\Lambda_{0}$ is Lagrangian, it's easy to check that $A$ is Hermitian. Now we can choose a suitable bases of $\mathbf{C}^{2 n} \oplus \mathbf{C}^{2 n}$ such that

$$
\Lambda_{0}=\left[\begin{array}{cc}
I_{k} & 0 \\
0 & A \\
0 & 0 \\
0 & I_{2 n-k}
\end{array}\right]
$$

under the symplectic form $J_{2 n}$. The left column corresponds to $\Lambda_{0} \cap \Lambda_{D}$ and the right column corresponds to $\left\{(A u, u)^{T} \mid u \in V\left(\Lambda_{0}\right)\right\}$. Let $\gamma(t)=\left[\begin{array}{ll}D_{1}(t) & D_{2}(t) \\ D_{3}(t) & D_{4}(t)\end{array}\right]$ be the fundamental solution of (1.5), then $\left[\begin{array}{cc}-I & 0 \\ D_{1} & D_{2} \\ 0 & I \\ D_{3} & D_{4}\end{array}\right]$ is a frame of $G r(\gamma)$ under the symplectic form $J_{2 n}$.

Next we will give a simple but useful lemma.

Lemma 2.6. For $M \in \operatorname{Sp}(2 n)$ and $\Lambda_{i} \in \operatorname{Lag}\left(\mathbf{C}^{2 n}, \omega\right)$ with $\omega(x, y)=\langle J x, y\rangle$, then $\Lambda_{i} \oplus \Lambda_{j}$ is a Lagrangian subspace of $\left(\mathbf{C}^{4 n},-\omega \oplus \omega\right)$ for $i, j=1, \cdots, 3$. We have

$$
\begin{gathered}
i\left(G r(M), \Lambda_{1} \oplus \Lambda_{2}, \Lambda_{1} \oplus \Lambda_{3}\right)=i\left(M \Lambda_{1}, \Lambda_{2}, \Lambda_{3}\right) . \\
i\left(G r(M), \Lambda_{1} \oplus \Lambda_{2}, \Lambda_{3} \oplus \Lambda_{2}\right)=i\left(M^{-1} \Lambda_{2}, \Lambda_{1}, \Lambda_{3},-\omega\right),
\end{gathered}
$$

where the triple index in the right of (2.15) is defined on $\left(\mathbf{C}^{2 n},-\omega\right)$.

Proof. We only give details to prove (2.14). In fact, for every $z=(u, M u)^{T} \in G r(M) \cap\left(\Lambda_{1} \oplus \Lambda_{2}+\Lambda_{1} \oplus \Lambda_{3}\right)$, there exist $u_{1}, v_{1} \in \Lambda_{1}, u_{2} \in \Lambda_{2}$ and $v_{3} \in \Lambda_{3}$ such that $z=z_{1}+z_{2}$, where $z_{1}=\left(u_{1}, u_{2}\right)^{T}, z_{2}=\left(v_{1}, v_{3}\right)^{T}$. It deduces $u=u_{1}+v_{1} \in \Lambda_{1}, M u=u_{2}+v_{3} \in M \Lambda_{1} \cap\left(\Lambda_{2}+\Lambda_{3}\right)$. Then by the definition (2.4), we have

$$
\mathfrak{Q}\left(G r(M), \Lambda_{1} \oplus \Lambda_{2} ; \Lambda_{1} \oplus \Lambda_{3}\right)(z, z)=\left\langle\mathcal{J} z_{1}, z_{2}\right\rangle=\left\langle-J u_{1}, v_{1}\right\rangle+\left\langle J u_{2}, v_{3}\right\rangle=\left\langle J u_{2}, v_{3}\right\rangle .
$$

Therefore, by definition (2.7) we have $\mathfrak{Q}\left(\operatorname{Gr}(M), \Lambda_{1} \oplus \Lambda_{2} ; \Lambda_{1} \oplus \Lambda_{3}\right)(z, z)=\mathfrak{Q}\left(M \Lambda_{1}, \Lambda_{2} ; \Lambda_{3}\right)(u, u)$ with $u \in M \Lambda_{1} \cap\left(\Lambda_{2}+\Lambda_{3}\right)$ and consequently we have

$$
m^{+}\left(\mathfrak{Q}\left(G r(M), \Lambda_{1} \oplus \Lambda_{2} ; \Lambda_{1} \oplus \Lambda_{3}\right)\right)=m^{+}\left(\mathfrak{Q}\left(M \Lambda_{1} \Lambda_{2} ; \Lambda_{3}\right)\right)
$$

Moreover, one can easily check that

$$
\begin{aligned}
\operatorname{dim}\left(G r(M) \cap\left(\Lambda_{1} \oplus \Lambda_{3}\right)\right) & =\operatorname{dim}\left(M \Lambda_{1} \cap \Lambda_{3}\right), \\
\operatorname{dim}\left(\operatorname{Gr}(M) \cap\left(\Lambda_{1} \oplus \Lambda_{2}\right) \cap\left(\Lambda_{1} \oplus \Lambda_{3}\right)\right) & =\operatorname{dim}\left(M \Lambda_{1} \cap \Lambda_{2} \cap \Lambda_{3}\right) .
\end{aligned}
$$

Thus by definition (2.7) and (2.16), (2.17) we get (2.14). The proof of (2.15) is totally similar and we omit the details. 
As a corollary of Lemma2.6, we have:

Corollary 2.7. For $M \in \operatorname{Sp}(2 n)$ and $\Lambda_{i} \in \operatorname{Lag}\left(\mathbf{C}^{2 n}, \omega\right)$ for $i=1, \cdots, 4$. We have

$$
i\left(G r(M), \Lambda_{1} \oplus \Lambda_{2}, \Lambda_{3} \oplus \Lambda_{4}\right)=i\left(M \Lambda_{1}, \Lambda_{2}, \Lambda_{4}\right)+i\left(M^{-1} \Lambda_{4}, \Lambda_{1}, \Lambda_{3},-\omega\right),
$$

and equivalently

$$
i\left(G r(M), \Lambda_{1} \oplus \Lambda_{2}, \Lambda_{3} \oplus \Lambda_{4}\right)=i\left(M \Lambda_{3}, \Lambda_{2}, \Lambda_{4}\right)+i\left(M^{-1} \Lambda_{2}, \Lambda_{1}, \Lambda_{3},-\omega\right) .
$$

Proof. From Lemma 2.6 and (2.10), we have

$$
\begin{aligned}
& i\left(G r(M), \Lambda_{1} \oplus \Lambda_{2}, \Lambda_{3} \oplus \Lambda_{4}\right)-i\left(G r(M), \Lambda_{1} \oplus \Lambda_{2}, \Lambda_{1} \oplus \Lambda_{4}\right) \\
& =s\left(G r(M), \Lambda_{1} \oplus \Lambda_{2} ; \Lambda_{1} \oplus \Lambda_{4}, \Lambda_{3} \oplus \Lambda_{4}\right) \\
& =i\left(G r(M), \Lambda_{1} \oplus \Lambda_{4}, \Lambda_{3} \oplus \Lambda_{4}\right)-i\left(\Lambda_{1} \oplus \Lambda_{2}, \Lambda_{1} \oplus \Lambda_{4}, \Lambda_{3} \oplus \Lambda_{4}\right) \\
& =i\left(M^{-1} \Lambda_{4}, \Lambda_{1}, \Lambda_{3},-\omega\right) .
\end{aligned}
$$

Since $i\left(\operatorname{Gr}(M), \Lambda_{1} \oplus \Lambda_{2}, \Lambda_{1} \oplus \Lambda_{4}\right)=i\left(M \Lambda_{1}, \Lambda_{2}, \Lambda_{4}\right)$, then we have (2.18). Similarly,

$$
\begin{aligned}
& i\left(G r(M), \Lambda_{1} \oplus \Lambda_{2}, \Lambda_{3} \oplus \Lambda_{4}\right)-i\left(G r(M), \Lambda_{1} \oplus \Lambda_{2}, \Lambda_{3} \oplus \Lambda_{2}\right) \\
& =s\left(G r(M), \Lambda_{1} \oplus \Lambda_{2} ; \Lambda_{3} \oplus \Lambda_{2}, \Lambda_{3} \oplus \Lambda_{4}\right) \\
& =i\left(G r(M), \Lambda_{3} \oplus \Lambda_{2}, \Lambda_{3} \oplus \Lambda_{4}\right)-i\left(\Lambda_{1} \oplus \Lambda_{2}, \Lambda_{3} \oplus \Lambda_{2}, \Lambda_{3} \oplus \Lambda_{4}\right) \\
& =i\left(M \Lambda_{3}, \Lambda_{2}, \Lambda_{4}\right) .
\end{aligned}
$$

Since $i\left(G r(M), \Lambda_{1} \oplus \Lambda_{2}, \Lambda_{3} \oplus \Lambda_{2}\right)=i\left(M^{-1} \Lambda_{2}, \Lambda_{1}, \Lambda_{3},-\omega\right)$, then we have (2.19). This complete the proof.

Example 2.8. Here we will give the precise computations of Example 1.7

1. [13, Theorem1.2] Recall that $V \subset \Lambda_{N}$ and $\Lambda_{V}=\mathcal{J} V^{\perp} \oplus V$. For every $x=\left(-u_{1}, u_{1}, v_{1}, v_{1}\right)^{T} \in$ $\operatorname{Gr}\left(I_{2 n}\right) \cap\left(\Lambda_{V}+\Lambda_{D}\right)$, there exist $y=\left(u_{2}, u_{3}, v_{2}, v_{3}\right)^{T} \in \Lambda_{V}$ and $z=\left(u_{4}, v_{4}, 0,0\right)^{T} \in \Lambda_{D}$ such that $x=y+z$ which derives $u_{2}+u_{3}=-\left(u_{4}+v_{4}\right), v_{1}=v_{2}=v_{3}$. By direct computations we have $\mathbb{Q}(x, x)=\omega(y, z)=0$. So $m^{+}\left(\mathfrak{Q}\left(\operatorname{Gr}\left(I_{2 n}\right), \Lambda_{V}, \Lambda_{D}\right)=0\right.$. Moreover, it's easy to check that $x=(-u, u, v, v)^{T} \in \operatorname{Gr}\left(I_{4 n}\right) \cap \Lambda_{V} \cap \Lambda_{D}$ if and only if $(-u, u)^{T} \in J_{2 n} V^{\perp}$ and $v=0$, then $\operatorname{dim}\left(\operatorname{Gr}\left(I_{2 n}\right) \cap \Lambda_{V} \cap \Lambda_{D}\right)=\operatorname{dim}\left(V^{\perp} \cap \operatorname{Gr}\left(-I_{n}\right)\right)$, where $\operatorname{Gr}\left(-I_{n}\right)=\left\{(u,-u), u \in \mathbf{C}^{n}\right\} \subset \Lambda_{N}$. Now we can conclude that

$$
\left.i\left(\operatorname{Gr}\left(I_{2 n}\right), \Lambda_{V}, \Lambda_{D}\right)=n-\operatorname{dim}\left(V^{\perp} \cap \operatorname{Gr}\left(-I_{n}\right)\right), \quad \Delta\left(\Lambda_{V}\right)=\operatorname{dim}\left(V^{\perp} \cap \operatorname{Gr}\left(-I_{n}\right)\right)\right) .
$$

Particularly, those two concrete cases considered in [13] Theorem 1.2] are very easy to be calculated directly.

\section{Separated boundary condition}

By 2.9) and 2.18), we have

$$
\begin{aligned}
i\left(\operatorname{Gr}\left(I_{2 n}\right), \Lambda_{s} \oplus \Lambda_{e}, \Lambda_{D}\right) & =i\left(\Lambda_{D}^{n}, \Lambda_{s}, \Lambda_{D}^{n},-\omega\right)+i\left(\Lambda_{s}, \Lambda_{e}, \Lambda_{D}^{n}\right) \\
& =n-\operatorname{dim}\left(\Lambda_{s} \cap \Lambda_{D}^{n}\right)+i\left(\Lambda_{s}, \Lambda_{e}, \Lambda_{D}^{n}\right),
\end{aligned}
$$

and consequently $\Delta\left(\Lambda_{s} \oplus \Lambda_{e}\right)=\operatorname{dim}\left(\Lambda_{s} \cap \Lambda_{D}^{n}\right)-i\left(\Lambda_{s}, \Lambda_{e}, \Lambda_{D}^{n}\right)$. Particularly, for the case where $y(0)=$ $A_{s} x(0), y(T)=A_{e} x(T)$, or equivalently, $\Lambda_{s}=\left\{(y, x)^{T} \mid y=A_{s} x\right\}, \Lambda_{e}=\left\{(y, x)^{T} \mid y=A_{e} x\right\}$, it's easy to check that $\Lambda_{s} \cap \Lambda_{D}^{n}=\{0\}$. Then by (2.7) we have 


$$
i\left(\Lambda_{s}, \Lambda_{e}, \Lambda_{D}^{n}\right)=m^{+}\left(\mathfrak{Q}\left(\Lambda_{s}, \Lambda_{e}, \Lambda_{D}^{n}\right)\right)
$$

In fact, for every $z=\left(A_{s} x, x\right)^{T} \in \Lambda_{s}=\Lambda_{e} \cap \Lambda_{D}^{n}$, there exist $y, u \in \mathbf{C}^{n}$ such that $\left[\begin{array}{c}A_{s} x \\ x\end{array}\right]=\left[\begin{array}{c}A_{e} y \\ y\end{array}\right]+\left[\begin{array}{c}u \\ 0\end{array}\right]$. Obviously, we have $x=y, u=A_{s} x-A_{e} y=\left(A_{s}-A_{e}\right) y$. Therefore,

$$
\mathfrak{Q}(z, z)=\left\langle\left[\begin{array}{cc}
0 & -I_{n} \\
I_{n} & 0
\end{array}\right]\left[\begin{array}{c}
A_{e} y \\
y
\end{array}\right],\left[\begin{array}{l}
u \\
0
\end{array}\right]\right\rangle=-\langle y, u\rangle=\left\langle\left(A_{e}-A_{s}\right) y, y\right\rangle .
$$

Consequently, there holds $m^{+}\left(\mathfrak{Q}\left(\Lambda_{s}, \Lambda_{e}, \Lambda_{D}^{n}\right)\right)=m^{+}\left(A_{e}-A_{s}\right)$. Now by (2.22) and (2.23), we have $i\left(\operatorname{Gr}\left(I_{2 n}\right), \Lambda_{s} \oplus\right.$ $\left.\Lambda_{e}, \Lambda_{D}\right)=n+m^{+}\left(A_{e}-A_{s}\right)$ and consequently $\Delta\left(G r\left(I_{2 n}\right), \Lambda_{s} \oplus \Lambda_{e}, \Lambda_{D}\right)=-m^{+}\left(A_{e}-A_{s}\right)$.

Next we will give a very simple concrete example to show how our result works for separated boundary condition.

Example 2.9. Let $\Lambda_{s}=\left\{(0, a)^{T} \mid a \in \mathbf{R}\right\}$ and $\Lambda_{e}=\{(b, b) \mid b \in \mathbf{R}\}$. Consider the Sturm-Liouville system

$$
-\ddot{x}(t)-x(t)=0,
$$

where $x \in W^{2,2}([0, \pi], \mathbf{R})$ and satisfies that $(\dot{x}(0), x(0))^{T} \in \Lambda_{s},(\dot{x}(\pi), x(\pi))^{T} \in \Lambda_{e}$.

The first step is to compute the Morse index. Consider the family of Sturm-Liouville system with parameter $s \in[0,+\infty)$ as

$$
-\ddot{x}(t)+(s-1) x(t)=0
$$

under the same boundary condition, by some direct computations we can conclude that there only exist $s_{1} \in$ $(0,1)$ and $s_{2} \in(1,+\infty)$ such that system (2.25) has solutions satisfying the boundary condition. Therefore the Morse index $m^{-}(x)=2$.

The second step is to compute the Maslov index. Let $y(t)=\dot{x}(t)$ and $z(t)=(y(t), x(t))^{T}$, then system (2.24) can be converted into Hamiltonian system (1.5) with $\mathcal{B}(t)=\left[\begin{array}{ll}1 & 0 \\ 0 & 1\end{array}\right]$. The fundamental solution is $\gamma(t)=\left[\begin{array}{cc}\cos t & -\sin t \\ \sin t & \cos t\end{array}\right]$. Then $\gamma(t) \Lambda_{s} \cap \Lambda_{e} \neq\{0\}$ if and only if $-\sin t=\cos t$. Note that there exists only one $t_{0} \in(0, \pi)$ such that $-\sin t_{0}=\cos t_{0}$ and the crossing form $\mathcal{B}\left(t_{0}\right)$ is positive definite, then by formula (2.2) the Maslov index $\mu^{-}(z)=1$. Therefore, there holds $\mu^{-}(z)-m^{-}(x)=-1$ which coincides with the formula (1.20).

\section{Morse index Theorem}

In order to prove the main theorem, we firstly introduce a theorem for the difference of Morse index of Hermitian form with its restriction on a subspace in $\$ 3.1$. We will prove our main results in $\$ 3.2$.

\subsection{An abstract difference Morse indices Theorem}

The following work is to introduce the definition of relative Morse index which will play an essential role in the proof of our main theorem. Let $X$ be a complex vector space and $\mathbb{Q}$ be a Hermitian form on $X$. For the general case please refer to [24, Section 3.3]. Let $V$ be a subspace of $X$ such that $\operatorname{dim} X / V<+\infty$. Let $V^{\mathfrak{Q}}$ be the space $\{x \in X \mid \mathbb{Q}(x, y)=0, \forall y \in V\}$. Denote $V^{\mathfrak{Q} Q}=\left(V^{\mathfrak{Q}}\right)^{\mathfrak{Q}}$. Generally, $\mathfrak{Q}$ induces an Hermitian form $\overline{\mathfrak{Q}}$ on 
$X / \operatorname{ker} \mathfrak{Q}$. Let $\mathcal{P}$ be the natural projection from $X$ to $X / \operatorname{ker} \mathfrak{Q}$. We have $\mathcal{P}\left(V^{\mathfrak{Q}}\right)=(\mathcal{P}(V))^{\mathfrak{Q}}$ for any subspace $V$. For a given subspace $V$ of $X$ such that $\operatorname{dim}(X / \operatorname{ker} \mathfrak{Q}) /(\mathcal{P} V))<+\infty$ and $V^{\mathfrak{Q} Q}=V+\operatorname{ker} \mathfrak{Q}$. We define the relative Morse index $I\left(\left.\mathfrak{Q}\right|_{V}, \mathfrak{Q}\right)$ as

$$
I\left(\left.\mathfrak{Q}\right|_{V}, \mathfrak{Q}\right)=\operatorname{dim}\left(\left(V \cap V^{\mathfrak{Q}}+\operatorname{ker} \mathfrak{Q}\right) / \operatorname{ker} \mathfrak{Q}\right)+m^{-}\left(\left.\mathfrak{Q}\right|_{V^{\mathfrak{Q}}}\right) .
$$

Theorem 3.1. If $V^{\mathfrak{Q}}=V+\operatorname{ker}(\mathfrak{Q})$ and both $m^{-}(\mathfrak{Q}), m^{-}\left(\left.\mathfrak{Q}\right|_{V^{\mathfrak{Q}}}\right.$ exist, then

$$
m^{-}(\mathfrak{Q})-m^{-}\left(\left.\mathfrak{Q}\right|_{V}\right)=I\left(\left.\mathfrak{Q}\right|_{V}, \mathfrak{Q}\right)
$$

Remark 3.2. In [4] Equation 1.2], there is an index theorem give by

$$
\operatorname{ind}(H)=\operatorname{ind}\left(\left.H\right|_{W}\right)+\operatorname{ind}\left(\left.H\right|_{W^{\perp}}\right)+\operatorname{dim}\left(W \cap W^{\perp}\right)-\operatorname{dim}(W \cap \operatorname{ker} H),
$$

where $H$ is a symmetric form on a finite dimensional real vector space $V$ (or a Hermitian form on a complex vector space) and $W \subset V$. Note that $\operatorname{dim}\left(\left(V \cap V^{\mathfrak{Q}}+\operatorname{ker} \mathfrak{Q}\right) / \operatorname{ker} \mathfrak{Q}\right)=\operatorname{dim}\left(V \cap V^{\mathfrak{Q}}\right)-\operatorname{dim}(V \cap \operatorname{ker} \mathfrak{Q})$, therefore, Theorem 3.1 can be viewed as a generalization of index form (3.3) to infinite dimensional situation.

In order to prove Theorem 3.1, we need several lemmas.

Lemma 3.3. Assume that $X=U+W$ and $\mathfrak{Q}(u, w)=0$ with $u \in U, w \in W$. We have $m^{-}(\mathfrak{Q})=m^{-}\left(\left.\mathfrak{Q}\right|_{U}\right)+$ $m^{-}\left(\left.\mathfrak{Q}\right|_{W}\right)$.

Proof. Let $U^{\prime}, W^{\prime}$ be maximum negative subspaces of $U, W$ respectively. Then for each $u \in U^{\prime}, w \in W^{\prime}$ we have $\mathfrak{Q}(u+w, u+w)=\mathfrak{Q}(u, u)+\mathfrak{Q}(w, w)<0$ if $u+w \neq 0$. Let $x \in U^{\prime} \cap W^{\prime}$. We have $\mathfrak{Q}(x, x)=0$. It follows that $U^{\prime} \cap W^{\prime}=0$. So $m^{-}(\mathfrak{Q}) \geq \operatorname{dim}\left(U^{\prime}+W^{\prime}\right)=\operatorname{dim}\left(U^{\prime}\right)+\operatorname{dim}\left(W^{\prime}\right)=m^{-}\left(\left.\mathfrak{Q}\right|_{U}\right)+m^{-}\left(\left.\mathfrak{Q}\right|_{W}\right)$.

Let $Y \supset U^{\prime}+W^{\prime}$ such that $\left.\mathfrak{Q}\right|_{Y}<0$. Let $y \in Y \cap\left(U^{\prime}+W^{\prime}\right)^{\mathfrak{Q}}$. Since $W \subset U^{\mathfrak{Q}} \subset U^{\prime \mathfrak{Q}}$, then $U^{\prime \mathfrak{Q}}=$ $U^{\prime 2} \cap(U+W)=U^{\prime 2} \cap U+W$. Similarly, we have $W^{\prime 2}=W^{\prime 2} \cap W+U$. It follows that $\left(U^{\prime}+W^{\prime}\right)^{2}=$ $U^{\prime 2} \cap W^{\prime 2}=\left(U^{\prime 2} \cap U+W\right) \cap\left(W^{\prime 2} \cap W+U\right)=\left(W^{\prime 2} \cap W\right)+\left(U^{\prime 2} \cap U+W\right) \cap U=\left(W^{\prime 2} \cap W\right)+\left(U^{\prime 2} \cap U\right)+(U \cap W)$. Then $y=y_{1}+y_{2}+y_{3}$ with $y_{1} \in U^{\prime 2} \cap U, y_{2} \in W^{\prime 2} \cap W, y_{3} \in W \cap U$.

Since $U^{\prime}, W^{\prime}$ are maximum negative subspaces of $U, W$ respectively, we have $\mathfrak{Q}\left(y_{1}, y_{1}\right) \geq 0, \mathfrak{Q}\left(y_{2}, y_{2}\right) \geq$ 0 . Note that $\mathfrak{Q}\left(y_{3}, y_{3}\right)=0$. We have $\mathfrak{Q}(y, y)=\mathfrak{Q}\left(y_{1}, y_{1}\right)+\mathfrak{Q}\left(y_{2}, y_{2}\right)+\mathfrak{Q}\left(y_{3}, y_{3}\right) \geq 0$. Note that $\mathfrak{Q}(y, y) \leq 0$ since $\left.\mathfrak{Q}\right|_{Y}<0$. Then we have $y=0$. It follows that $Y=U^{\prime}+W^{\prime}$. Then we have

$$
m^{-}(\mathfrak{Q})=\operatorname{dim}\left(U^{\prime}+W^{\prime}\right)=m^{-}\left(\left.\mathfrak{Q}\right|_{U}\right)+m^{-}\left(\left.\mathfrak{Q}\right|_{W}\right) .
$$

Lemma 3.4. If $\operatorname{dim} X / V<+\infty$, then

$$
\operatorname{dim} V^{\mathfrak{Q}} / \operatorname{ker} \mathfrak{Q}=\operatorname{dim}\left(X / V^{\mathbb{Q}}\right) .
$$

If $\operatorname{dim} W<+\infty$, then

$$
\operatorname{dim} X / W^{\mathfrak{Q}}=\operatorname{dim}(W+\operatorname{ker} \mathfrak{Q}) / \operatorname{ker} \mathfrak{Q} .
$$

Proof. Consider the sesquilinear forms $\tilde{\mathfrak{Q}}: X / V \times V^{\mathfrak{Q}} \rightarrow \mathbf{C}$ such that $\tilde{\mathfrak{Q}}(x+V, u)=\mathfrak{Q}(x, u)$ with $x+V \in$ $X / V, u \in V^{Q}$. It is well-defined and it induces a finite dimensional linear map $A: X / V \rightarrow V^{Q}$ such that 
$\tilde{\mathfrak{Q}}(p, q)=(A p, q)$ with $p \in X / V, q \in V^{\mathfrak{Q}}$. Note that $\operatorname{ker} A=V^{\mathbb{Q} Q} / V$ and $\operatorname{ker} A^{*}=\operatorname{ker} \mathfrak{Q}$. Then we have $\operatorname{dim} X / V-\operatorname{dim} V^{\mathfrak{Q} Q} / V=\operatorname{dim} V^{\mathfrak{Q}}-\operatorname{dim} \operatorname{ker} \mathfrak{Q}$. It follows that

$$
\operatorname{dim} X /(V+\operatorname{ker} \mathfrak{Q})=\operatorname{dim} X / V^{\mathfrak{Q}}=\operatorname{dim} V^{\mathfrak{Q}} / \operatorname{ker} \mathfrak{Q} .
$$

Similarly if we consider sesquilinear form on $X / W^{\mathbb{Q}} \times W$, then we get

$$
\operatorname{dim} X / W^{\mathfrak{Q}}=\operatorname{dim} W /(W \cap \operatorname{ker} \mathfrak{Q})=\operatorname{dim}(W+\operatorname{ker} \mathfrak{Q}) / \operatorname{ker} \mathfrak{Q}
$$

Lemma 3.5. Assume that $V^{\mathbb{Q}}=V$, $\operatorname{dim} X / V<+\infty, V^{\mathbb{Q}} \subset V$. Then we have

$$
m^{-}(\mathfrak{Q})-m^{-}\left(\left.\mathfrak{Q}\right|_{V}\right)=\operatorname{dim}\left(V^{\mathfrak{Q}} / \operatorname{ker} \mathfrak{Q}\right)
$$

Proof. There is a finite dimensional linear subspace $W \subset X$ such that $X=V \oplus W$. Then we have $\left(W^{\mathbb{Q}} \cap V^{\mathbb{Q}}\right)=$ $(W+V)^{\mathfrak{Q}}=\operatorname{ker} \mathfrak{Q}$. Note that $\operatorname{ker} \mathfrak{Q} \subset V^{\mathfrak{Q}} \subset V$. We have $W \cap \operatorname{ker} \mathfrak{Q}=\{0\}$. Then by Lemma 3.4, we have $\operatorname{dim}\left(X / W^{\mathfrak{Q}}\right)=\operatorname{dim} W$ and $\operatorname{dim} X / V=\operatorname{dim} V^{\mathbb{Q}} / \operatorname{ker} \mathfrak{Q}$. It follows that

$$
\begin{aligned}
\operatorname{dim} X /\left(V^{\mathfrak{Q}}+W^{\mathfrak{Q}}\right) & =\operatorname{dim} X / W^{\mathfrak{Q}}-\operatorname{dim}\left(V^{\mathfrak{Q}}+W^{\mathfrak{Q}}\right) / W^{\mathfrak{Q}}=\operatorname{dim} W-\operatorname{dim} V^{\mathfrak{Q}} /\left(W^{\mathfrak{Q}} \cap V^{\mathfrak{Q}}\right) \\
& =\operatorname{dim} W-\operatorname{dim} V^{\mathfrak{Q}} / \operatorname{ker} \mathfrak{Q}=\operatorname{dim} X / V-\operatorname{dim} X / V=0 .
\end{aligned}
$$

It follows that $X=V^{\mathbb{Q}}+W^{\mathbb{Q}}$. Then we have $V=\left(V^{\mathbb{Q}}+W^{\mathbb{Q}}\right) \cap V=V^{\mathbb{Q}}+\left(V \cap W^{\mathbb{Q}}\right)$ and $X=V+W=$ $V^{\mathfrak{Q}}+W+\left(V \cap W^{\mathfrak{Q}}\right)$. Note that $\left(V \cap W^{\mathfrak{Q}}\right)^{\mathfrak{Q}} \supset\left(V^{\mathfrak{Q}}+W^{\mathfrak{Q}}\right)$. By Lemma 3.3, we have $m^{-}\left(\left.\mathfrak{Q}\right|_{V}\right)=m^{-}\left(\left.\mathfrak{Q}\right|_{W^{\mathfrak{Q}} \cap V}\right)+$ $m^{-}\left(\left.\mathfrak{Q}\right|_{V^{\mathfrak{Q}}}\right)$, and $m^{-}(\mathfrak{Q})=m^{-}\left(\left.\mathfrak{Q}\right|_{W^{\mathfrak{Q}} \cap V}\right)+m^{-}\left(\left.\mathfrak{Q}\right|_{V^{\mathfrak{a}}+W}\right)$. Since $V^{\mathfrak{Q}} \subset V$, then $\left.\mathfrak{Q}\right|_{V^{\mathfrak{Q}}}=0$, and it follows that $m^{-}\left(\left.\mathfrak{Q}\right|_{V}\right)=m^{-}\left(\left.\mathfrak{Q}\right|_{W^{2} \cap V}\right)$.

Let $\tilde{Q}=\left.\mathfrak{Q}\right|_{V^{\mathbb{Q}}+W}$. Then ker $\tilde{Q}=\operatorname{ker} \mathfrak{Q} \subset V^{\mathbb{Q}}$. Since $V^{\mathfrak{Q}} \subset V$, we have $\left.\mathfrak{Q}\right|_{V^{\mathfrak{Q}}}=0$. It follows that $m^{ \pm}(\tilde{\mathfrak{Q}}) \geq \operatorname{dim}\left(V^{\mathfrak{Q}} / \operatorname{ker} \mathfrak{Q}\right)$. Let $k=\operatorname{dim} W$. Since $\operatorname{dim} V^{\mathfrak{Q}} / \operatorname{ker} \mathfrak{Q}=\operatorname{dim} W$, we have $2 k \leq m^{-}(\tilde{\mathfrak{Q}})+m^{+}(\tilde{\mathfrak{Q}}) \leq$ $\operatorname{dim}\left(V^{\mathfrak{Q}}+W\right) / \operatorname{ker} \mathfrak{Q}=2 k$. It follows that $m^{-}(\tilde{\mathbb{Q}})=k=\operatorname{dim}\left(V^{\mathfrak{Q}} / \operatorname{ker} \mathbb{Q}\right)$.

Then we can prove Theorem 3.1

Proof of Theorem 3.1] Let $W=V+V^{\mathfrak{Q}}$. Then there is a linear subspace $U \subset V^{\mathfrak{Q}}$ such that $W=V \oplus U$. Since $\operatorname{dim}(X / V)<+\infty$, we see that $\operatorname{dim} U<\infty$. For each $x \in V, y \in U \subset V^{\mathbb{Q}}$, we have $\mathfrak{Q}(x, y)=0$. It follows that $m^{-}\left(\left.\mathfrak{Q}\right|_{W}\right)-m^{-}\left(\left.\mathfrak{Q}\right|_{V}\right)=m^{-}(U)$. Note that $V^{\mathfrak{Q}}=V^{\mathfrak{Q}} \cap(U+V)=\left(V \cap V^{\mathfrak{Q}}\right) \oplus U$. Since $V^{\mathfrak{Q}} \cap V \subset V^{\mathbb{Q}}$, we

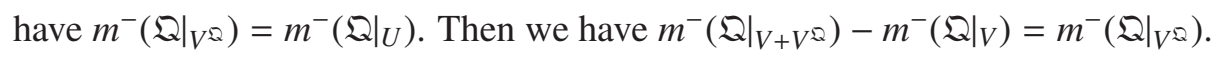

Since $V^{\mathbb{Q}}=V+\operatorname{ker} \mathbb{Q}$, we have $V+V^{\mathbb{Q}}=V^{\mathbb{Q}}+V^{\mathbb{Q}}$. By Lemma 3.4 we have

$$
\begin{aligned}
\operatorname{dim} X /\left(V^{\mathbb{Q}}+V^{\mathbb{Q}}\right) & =\operatorname{dim}\left(X / V^{\mathbb{Q}}\right)-\operatorname{dim}\left(V^{\mathbb{Q}}+V^{\mathbb{Q}}\right) / V^{\mathbb{Q}}=\operatorname{dim} V^{\mathbb{Q}} / \operatorname{ker} \mathbb{Q}-\operatorname{dim} V^{\mathbb{Q}} /\left(V^{\mathbb{Q}} \cap V^{\mathbb{Q}}\right) \\
& =\operatorname{dim}\left(V^{\mathbb{Q}} \cap V^{\mathbb{Q}}\right) / \operatorname{ker} \mathbb{Q}=\operatorname{dim}\left(X /\left(V^{\mathbb{Q}}+V^{\mathbb{Q}}\right)^{\mathbb{Q}}\right) .
\end{aligned}
$$

It follows that $\left(V+V^{\mathbb{Q}}\right)^{\mathbb{Q}}=V+V^{\mathbb{Q}}$. Then by Lemma 3.5, we have

$$
m^{-}(\mathfrak{Q})-m^{-}\left(\left.\mathfrak{Q}\right|_{V+V^{\mathfrak{Q}}}\right)=\operatorname{dim}\left(V^{\mathfrak{Q} Q} \cap V^{\mathfrak{Q}}\right) / \operatorname{ker} \mathfrak{Q}=\operatorname{dim}\left(V \cap V^{\mathfrak{Q}}+\operatorname{ker} \mathfrak{Q}\right) / \operatorname{ker} \mathfrak{Q}
$$


Lemma 3.6. We assume that $X$ is a Hilbert space, $H$ is a closed subspace of $X$, and $\mathfrak{Q}(x, y)=(A x, y)$ with bounded Fredholm self-adjoint operator A. Then we have

$$
H^{\mathbb{Q}}=H+\operatorname{ker} \mathfrak{Q}
$$

Proof. Since $A$ is a Fredholm self-adjoint operator, then $\operatorname{dim} \operatorname{ker} A<\infty, \operatorname{ker} A=(\operatorname{Im} A)^{\perp}$ and $\operatorname{ker} A=\operatorname{ker} \mathfrak{Q}$. Since $(A x, y)=(x, A y)$, we have $H^{\mathfrak{Q}}=(A H)^{\perp}=A^{-1}\left(H^{\perp}\right)=A^{-1}\left(H^{\perp} \cap \operatorname{Im} A\right)$. It follows that

$$
H^{\mathfrak{Q}}=\left(A\left(A^{-1}\left(H^{\perp} \cap \operatorname{Im} A\right)\right)\right)^{\perp}=\left(H^{\perp} \cap \operatorname{Im} A\right)^{\perp}=\overline{H^{\perp \perp}+\operatorname{Im} A^{\perp}}=\overline{H^{\perp \perp}+\operatorname{ker} A} .
$$

Since $H$ is closed and $\operatorname{dim} \operatorname{ker} A<+\infty$, then $H^{\perp \perp}=H$ and $(H+\operatorname{ker} A)$ is closed. Then we have

$$
H^{\mathbb{Q}}=\overline{H^{\perp \perp}+\operatorname{ker} A}=H+\operatorname{ker} \mathbb{Q} .
$$

\subsection{Morse index Theorem}

Let $A$ be the Hermitian matrix defined by $\Lambda_{0}$. For a stationary point $x$ of system 1.1 , its index form $I_{x}$ is given by

$$
I_{x}(\xi, \eta)=\int_{0}^{T}\left\{\langle P \dot{\xi}, \dot{\eta}\rangle+\langle Q \xi, \dot{\eta}\rangle+\left\langle Q^{T} \dot{\xi}, \eta\right\rangle+\langle R \xi, \eta\rangle\right\} d t-\left\langle A\left[\begin{array}{c}
\xi(0) \\
\xi(T)
\end{array}\right],\left[\begin{array}{l}
\eta(0) \\
\eta(T)
\end{array}\right]\right\rangle
$$

on

$$
H_{\Lambda_{0}}([0, T])=\left\{\xi \in W^{1,2}\left([0, T], \mathbf{C}^{n}\right) \mid(\xi(0), \xi(T)) \in V\left(\Lambda_{0}\right)\right\} .
$$

For convenience, we will drop the subscript $x$ of $I_{x}$. By [18, Theorem A2], the space $W^{1,2}(0, T)$ can be continuously imbedded into the space

$$
\mathbf{C}^{0}=\left\{u \in \mathbf{C}^{0}(0, T)\left|\|u\|_{\mathbf{C}^{0}}=\sup _{0<t<T}\right| u(t) \mid<\infty\right\}
$$

and for any $\varepsilon>0$, there exists a constant $C_{\varepsilon}$ such that

$$
\|u\|_{\mathbf{C}^{0}} \leq \varepsilon\|\dot{u}\|_{L^{2}(0, T)}+C_{\varepsilon}\|u\|_{L^{2}(0, T)}
$$

By (3.7) and (3.8) we have

$$
\begin{aligned}
I(\xi, \xi) & \geq c_{1}\|\dot{\xi}\|_{L^{2}}^{2}+c_{2}\|\xi\|_{L^{2}}\|\dot{\xi}\|_{L^{2}}+c_{3}\|\xi\|_{L^{2}}^{2}-\varepsilon_{1}\|\dot{\xi}\|_{L^{2}}^{2}-C_{\varepsilon_{1}}\|\xi\|_{L^{2}}^{2} \\
& \geq c_{1}\|\dot{\xi}\|_{L^{2}}^{2}-\varepsilon_{2} c_{2}\|\dot{\xi}\|_{L^{2}}^{2}-C_{\varepsilon_{2}} c_{2}\|\xi\|_{L^{2}}^{2}+c_{3}\|\xi\|_{L^{2}}^{2}-\varepsilon_{1}\|\dot{\xi}\|_{L^{2}}^{2}-C_{\varepsilon_{1}}\|\xi\|_{L^{2}}^{2} \\
& =c_{4}\left(\|\dot{\xi}\|_{L^{2}}^{2}+\|\xi\|_{L^{2}}^{2}\right)+c_{5}\|\xi\|_{L^{2}}^{2}
\end{aligned}
$$

where $c_{i}, i=1, \ldots, 5$ and $\varepsilon_{i}, C_{\varepsilon_{i}}, i=1,2$ are constants such that $c_{1}, c_{4}>0, \varepsilon_{i}$ is small enough. Moreover, the second inequality comes from Young inequality. Similarly, for some suitable constants $C_{4}, C_{5}$ we have

$$
I(\xi, \xi) \leq C_{4}\left(\|\dot{\xi}\|_{L^{2}}^{2}+\|\xi\|_{L^{2}}^{2}\right)+C_{5}\|\xi\|_{L^{2}}^{2} .
$$

Then we can choose a constant $C_{H_{\Lambda_{0}}}$ large enough such that $I(\xi, \xi)+C_{H_{\Lambda_{0}}}\|\xi\|_{L^{2}}^{2}$ is a norm equivalent with the $W^{1,2}$-norm on $H_{\Lambda_{0}}([0, T])$ and will be denoted by $\|\cdot\|_{H_{\Lambda_{0}}}$. 
Recall the definition of operator $\mathcal{A}$ in (1.3) and integral by parts, then for every $\xi \in E_{\Lambda_{0}}(0, T)$ and $\eta \in H_{\Lambda_{0}}([0, T])$ we have

$$
\begin{aligned}
\langle\mathcal{A} \xi, \eta\rangle_{L^{2}} & =\int_{0}^{T}\left\langle-\frac{d}{d t}(P \dot{\xi}+Q \xi)+Q^{T} \dot{\xi}+R \xi, \eta\right\rangle d t \\
& =\int_{0}^{T}\left\{\langle P \dot{\xi}, \dot{\eta}\rangle+\langle Q \xi, \dot{\eta}\rangle+\left\langle Q^{T} \dot{\xi}, \eta\right\rangle+\langle R \xi, \eta\rangle\right\} d t-\left\langle\left[\begin{array}{c}
-y(0) \\
y(T)
\end{array}\right],\left[\begin{array}{c}
\eta(0) \\
\eta(T)
\end{array}\right]\right\rangle \\
& =\int_{0}^{T}\left\{\langle P \dot{\xi}, \dot{\eta}\rangle+\langle Q \xi, \dot{\eta}\rangle+\left\langle Q^{T} \dot{\xi}, \eta\right\rangle+\langle R \xi, \eta\rangle\right\} d t-\left\langle A\left[\begin{array}{c}
\xi(0) \\
\xi(T)
\end{array}\right],\left[\begin{array}{c}
\eta(0) \\
\eta(T)
\end{array}\right]\right\rangle \\
& =I(\xi, \eta),
\end{aligned}
$$

where $y(t)=P(t) \dot{\xi}(t)+Q(t) \xi(t)$. The third equation holds since $\xi$ satisfies the boundary condition $\Lambda_{0}$ in the form (2.13), therefore we can decompose $\left[\begin{array}{c}-y(0) \\ y(T)\end{array}\right]=\left[\begin{array}{c}-y_{1}(0) \\ y_{1}(T)\end{array}\right]+\left[\begin{array}{c}-y_{2}(0) \\ y_{2}(T)\end{array}\right]$ such that $\left(-y_{1}(0), y_{1}(T)\right)^{T} \in J_{2 n} V^{\perp}$ and $\left(-y_{2}(0), y_{2}(T)\right)^{T}=A(\xi(0), \xi(T))^{T} \in J_{2 n} V$.

Let $m^{-}\left(I, \Lambda_{0}\right)$ be the dimension of the maximal negative definite subspace of the index form $I$ on $H_{\Lambda_{0}}([0, T])$. The following theorem is standard, but for reader's convenience, we give details of the proof.

Theorem 3.7. For a critical point $x$ of the Lagrangian system (1.1) with the boundary condition (1.4),

$$
\operatorname{dim} \operatorname{ker}(I)=m^{0}(\mathcal{A}), \quad m^{-}\left(I, \Lambda_{0}\right)=m^{-}(\mathcal{A}) .
$$

Proof. For the first equation we only need to prove that $\xi \in \operatorname{ker} I$ is equivalent to $\xi \in \operatorname{ker} \mathcal{A}$.

$\Leftarrow$ It's obvious by (3.11).

$\Rightarrow \quad$ Assuming that $\xi \in \operatorname{ker}(I)$, then $I(\xi, \eta)=0$ for any $\eta \in H_{\Lambda_{0}}([0, T])$. So for every $\eta \in H_{\Lambda_{0}}([0, T])$ such that $\eta(0)=\eta(T)=0$, by 3.7 we have

$$
\begin{aligned}
0=I(\xi, \eta) & =\int_{0}^{T}\left\{\langle P \dot{\xi}, \dot{\eta}\rangle+\langle Q \xi, \dot{\eta}\rangle+\left\langle Q^{T} \dot{\xi}, \eta\right\rangle+\langle R \xi, \eta\rangle\right\} d t-\left\langle A\left[\begin{array}{c}
\xi(0) \\
\xi(T)
\end{array}\right],\left[\begin{array}{l}
\eta(0) \\
\eta(T)
\end{array}\right]\right\rangle \\
& =\int_{0}^{T}\langle P \dot{\xi}+Q \xi, \dot{\eta}\rangle d t+\int_{0}^{T}\left\langle-\int_{0}^{t}\left(Q^{T} \dot{\xi}+R \xi\right) d s, \dot{\eta}\right\rangle d t \\
& =\int_{0}^{T}\left\langle P \dot{\xi}+Q \xi-\int_{0}^{t}\left(Q^{T} \dot{\xi}+R \xi\right) d s, \dot{\eta}\right\rangle d t .
\end{aligned}
$$

Then by $d u$ Bois - Reymond theorem we have

$$
P \dot{\xi}+Q \xi-\int_{0}^{t}\left(Q^{T} \dot{\xi}+R \xi\right) d s=\text { constant }
$$

Derivative on both sides of (3.14) leads that $\xi$ is a solution of (1.3). The rest is to prove $x$ satisfies condition (1.4). Decomposing $\left[\begin{array}{c}-y(0) \\ y(T)\end{array}\right]$ into $\left[\begin{array}{c}-y(0) \\ y(T)\end{array}\right]=\left[\begin{array}{c}-y_{1}(0) \\ y_{1}(T)\end{array}\right]+\left[\begin{array}{c}-y_{2}(0) \\ y_{2}(T)\end{array}\right]$, where $\left[\begin{array}{c}-y_{1}(0) \\ y_{1}(T)\end{array}\right] \in J_{2 n} V^{\perp}$ and $\left[\begin{array}{c}-y_{2}(0) \\ y_{2}(T)\end{array}\right] \in$ $J_{2 n} V$, then by (3.7) we have

$$
I(x, \eta)=\left\langle\left[\begin{array}{c}
-y_{1}(0) \\
y_{1}(T)
\end{array}\right]+\left[\begin{array}{c}
-y_{2}(0) \\
y_{2}(T)
\end{array}\right]-A\left[\begin{array}{c}
\xi(0) \\
\xi(T)
\end{array}\right],\left[\begin{array}{c}
\eta(0) \\
\eta(T)
\end{array}\right]\right\rangle .
$$


Since $\eta \in H_{\Lambda_{0}}([0, T])$ is arbitrary, then we can take $\eta$ such that $\left[\begin{array}{c}-y_{2}(0) \\ y_{2}(T)\end{array}\right]-A\left[\begin{array}{c}\xi(0) \\ \xi(T)\end{array}\right]=\left[\begin{array}{l}\eta(0) \\ \eta(T)\end{array}\right]$, then $\left[\begin{array}{c}-y_{2}(0) \\ y_{2}(T)\end{array}\right]-$ $A\left[\begin{array}{l}\xi(0) \\ \xi(T)\end{array}\right]=0$. This means the condition (1.4) holds.

In order to prove the second equation we decompose $E_{\Lambda_{0}}(0, T)$ into $E^{+} \oplus E^{0} \oplus E^{-}$, where $E^{+}, E^{0}$ and $E^{-}$ are the eigenspaces of $\mathcal{A}$ in $E_{\Lambda_{0}}(0, T)$ corresponding to the positive, zero, negative eigenvalues respectively. For every eigenvector $\eta$ corresponding to a negative eigenvalue $\lambda$ of $\mathcal{A}$, by (3.11) we have

$$
I(\eta, \eta)=\langle\mathcal{A} \eta, \eta\rangle=\langle\lambda \eta, \eta\rangle<0 .
$$

Therefore, $m^{-}\left(I, \Lambda_{0}\right) \geq m^{-}(\mathcal{A})$.

Let $H_{\Lambda_{0}}([0, T])^{-}$be the maximal negative subspace of index form $I$ and $\left\{e_{1}, \ldots, e_{k}\right\}$ be the orthogonal bases of $E^{-}$and $\left\{e_{k+1}, e_{k+2}, \ldots\right\}$ be the orthogonal bases of $E^{0} \oplus E^{+}$. If $\xi \in H_{\Lambda_{0}}([0, T])$ satisfies that

$$
\begin{aligned}
0 & =\left\langle e_{i}, \xi\right\rangle_{H_{\Lambda_{0}}}=I\left(e_{i}, \xi\right)+C_{H_{\Lambda_{0}}}\left\langle e_{i}, \xi\right\rangle_{L^{2}} \\
& =\left\langle\mathcal{A} e_{i}, \xi\right\rangle_{L^{2}}+C_{H_{\Lambda_{0}}}\left\langle e_{i}, \xi\right\rangle_{L^{2}}=\left(\lambda_{i}+C_{H_{\Lambda_{0}}}\right)\left\langle e_{i}, \xi\right\rangle_{L^{2}}, \quad \forall e_{i},
\end{aligned}
$$

where $\langle\cdot, \cdot\rangle_{H_{\Lambda_{0}}}$ is the inner product induced by $\|\cdot\|_{H_{\Lambda_{0}}}$, then there must hold $\xi=0$. Therefore, we have

$$
\operatorname{span}\left\{e_{1}, \ldots, e_{k}\right\} \oplus \overline{\operatorname{span}\left\{e_{k+1}, e_{k+2}, \ldots\right\}}=H_{\Lambda_{0}}([0, T])
$$

Then if $m^{-}\left(I, \Lambda_{0}\right)>m^{-}(\mathcal{A})$, there must exist $\xi \neq 0 \in H_{\Lambda_{0}}([0, T])^{-} \cap\left(E^{0} \oplus E^{+}\right)$. But by (3.7) we have $I(\xi, \xi) \geq 0$ which is a contradiction. Therefore there must hold $m^{-}\left(I, \Lambda_{0}\right)=m^{-}(\mathcal{A})$. We conclude the proof.

Now we will give the direct proof of Theorem 1.1

Proof of Theorem 1.1. Let $H_{0}=\left\{\xi \in W^{1,2}\left([0, T], \mathbf{C}^{n}\right) \mid \xi(0)=\xi(T)=0\right\}$ and $H_{0}^{I}=\{\xi \in H \mid I(\xi, \eta)=0, \forall \eta \in$ $\left.H_{0}\right\}$. Obviously, if $\xi \in W^{2,2}\left([0, T], \mathbf{C}^{n}\right)$, then integral by parts of index form (3.7), we have

$$
I(\xi, \eta)=\int_{0}^{T}\left\langle-\frac{d}{d t}(P \dot{\xi}+Q \xi)+Q^{T} \dot{\xi}+R \xi, \eta\right\rangle d t+\left\langle\left[\begin{array}{c}
-y(0) \\
y(T)
\end{array}\right]-A\left[\begin{array}{c}
\xi(0) \\
\xi(T)
\end{array}\right],\left[\begin{array}{l}
\eta(0) \\
\eta(T)
\end{array}\right]\right\rangle,
$$

where $y(t)=P(t) \dot{\xi}(t)+Q(t) \xi(t)$. Actually, $H_{0}^{I}$ can be expressed in a specific way by

$$
H_{0}^{I}=\left\{\xi \in H \mid-\frac{d}{d t}(P(t) \dot{\xi}(t)+Q(t) \xi(t))+Q(t)^{T} \dot{\xi}(t)+R(t) \xi(t)=0\right\} .
$$

Recall the discussions in (3.9), 3.10), we can choose a constant $C_{H_{\Lambda_{0}}}$ large enough such that $I(\xi, \xi)+$ $C_{H_{\Lambda_{0}}}\|\xi\|_{L^{2}}^{2}$ is a norm equivalent with the $W^{1,2}$-norm on $H_{\Lambda_{0}}([0, T])$ and will be denoted by $\|\cdot\|_{H_{\Lambda_{0}}}$. Moreover, we will denote $\langle\cdot, \cdot\rangle_{H_{\Lambda_{0}}}$ the inner product induced by $\|\cdot\|_{H_{\Lambda_{0}}}$. Note that the injection from $W^{1,2}(0, T)$ to $L^{2}(0, T)$ is compact, then for every $\xi, \eta \in H_{\Lambda_{0}}$, we have $I(\xi, \eta)=\langle\xi, \eta\rangle_{H_{\Lambda_{0}}}-C_{H_{\Lambda_{0}}}\|\xi\|_{L^{2}}^{2}=\langle(\mathcal{I}+\mathcal{T}) \xi, \eta\rangle_{H_{\Lambda_{0}}}$ with compact operator $\mathcal{T}$. Obviously, $\mathcal{I}+\mathcal{T}$ is a bounded self-adjoint Fredholm operator. Since $H_{0}$ is a close subspace of $H_{\Lambda_{0}}([0, T])$, then by Lemma 3.4 we have $H_{0}^{I I}=H_{0}+\operatorname{ker} I$. By Theorem 3.1 we have

$$
m^{-}\left(\Lambda_{0}\right)-m^{-}\left(\Lambda_{D}\right)=m^{-}\left(\left.I\right|_{H_{0}^{I}}\right)+\operatorname{dim}\left(\left(H_{0} \cap H_{0}^{I}+\operatorname{ker} I\right) / \operatorname{ker} I\right) .
$$


For every $\xi \in H_{0}^{I}$, let $p=\left[\begin{array}{c}-y(0) \\ y(T)\end{array}\right], q=\left[\begin{array}{l}\xi(0) \\ \xi(T)\end{array}\right]$ and $z=\left[\begin{array}{l}p \\ q\end{array}\right]$, then by $(3.16)$ we have

$$
\left.I(\xi, \xi)=\langle p-A q, q\rangle=\left\langle\begin{array}{cc}
0 & -I_{2 n} \\
I_{2 n} & 0
\end{array}\right]\left[\begin{array}{c}
p-A q \\
0
\end{array}\right],\left[\begin{array}{l}
p \\
q
\end{array}\right]\right\rangle .
$$

Note that $\left[\begin{array}{l}p \\ q\end{array}\right] \in G r(\gamma(T)) \cap\left(\Lambda_{0}+\Lambda_{D}\right)$. So there exists some $p_{1} \in J_{2 n} V^{\perp}$ such that $\left[\begin{array}{c}p_{1}+A q \\ q\end{array}\right] \in \Lambda_{0}$ and the split $\left[\begin{array}{l}p \\ q\end{array}\right]=\left[\begin{array}{c}p_{1}+A q \\ q\end{array}\right]+\left[\begin{array}{c}p-p_{1}-A q \\ 0\end{array}\right]$ holds. Since $\left.\left\langle\begin{array}{cc}0 & -I_{2 n} \\ I_{2 n} & 0\end{array}\right]\left[\begin{array}{c}-p_{1} \\ 0\end{array}\right],\left[\begin{array}{l}p \\ q\end{array}\right]\right\rangle=0$, then by the definition of triple index there holds

$$
I(\xi, \xi)=\left\langle\left[\begin{array}{cc}
0 & -I_{2 n} \\
I_{2 n} & 0
\end{array}\right]\left[\begin{array}{c}
p-A q-p_{1} \\
0
\end{array}\right],\left[\begin{array}{l}
p \\
q
\end{array}\right]\right\rangle=-\mathfrak{Q}(z, z)
$$

and therefore we have

$$
m^{-}\left(\left.I\right|_{H_{0}^{I}}\right)=m^{-}\left(-\mathfrak{Q}\left(G r\left(\gamma(T), \Lambda_{0} ; \Lambda_{D}\right)\right)=m^{+}\left(\mathfrak{Q}\left(G r\left(\gamma(T), \Lambda_{0} ; \Lambda_{D}\right)\right) .\right.\right.
$$

Note that

$$
\begin{aligned}
\operatorname{dim}\left(\left(H_{0} \cap H_{0}^{I}+\operatorname{ker} I\right) / \operatorname{ker} I\right) & =\operatorname{dim}\left(H_{0} \cap H_{0}^{I}\right)-\operatorname{dim}\left(H_{0} \cap H_{0}^{I} \cap \operatorname{ker} I\right) \\
& =\operatorname{dim}\left(G r(\gamma(T)) \cap \Lambda_{0}\right)-\operatorname{dim}\left(G r(\gamma(T)) \cap \Lambda_{0} \cap \Lambda_{D}\right),
\end{aligned}
$$

then by (2.8), (3.17), (3.18) and (3.19), we have

$$
m^{-}\left(\Lambda_{0}\right)-m^{-}\left(\Lambda_{D}\right)=i\left(G r(\gamma(T)), \Lambda_{0}, \Lambda_{D}\right)
$$

This complete the proof.

Now we can present the proof of Corollary 1.6

Proof of Corollary 1.6 Denote the Maslov index for Dirichlet boundary condition by $\mu_{d}^{-}(z)$, then as wellknown that (for example [13, Remark 3.6] ) $\mu_{d}^{-}(z)-m^{-}\left(\Lambda_{D}\right)=n$. By direct computations, we have

$$
\begin{aligned}
m^{-}(x)-\mu^{-}(z) & =\left(m^{-}(x)-m^{-}\left(\Lambda_{D}\right)\right)+\left(m^{-}\left(\Lambda_{D}\right)-\mu_{d}^{-}(z)\right)+\left(\mu_{d}^{-}(z)-\mu^{-}(z)\right) \\
& =i\left(\operatorname{Gr}(\gamma(T)), \Lambda_{0}, \Lambda_{D}\right)-n+s\left(\operatorname{Gr}\left(I_{2 n}\right), \operatorname{Gr}(\gamma(T)) ; \Lambda_{0}, \Lambda_{D}\right) \\
& =i\left(\operatorname{Gr}(\gamma(T)), \Lambda_{0}, \Lambda_{D}\right)-n+i\left(\operatorname{Gr}\left(I_{2 n}\right), \Lambda_{0}, \Lambda_{D}\right)-i\left(\operatorname{Gr}(\gamma(T)), \Lambda_{0}, \Lambda_{D}\right) \\
& =-n+i\left(\operatorname{Gr}\left(I_{2 n}\right), \Lambda_{0}, \Lambda_{D}\right) .
\end{aligned}
$$

This means $\mu^{-}(z)-m^{-}(x)=n-i\left(G r\left(I_{2 n}\right), \Lambda_{0}, \Lambda_{D}\right)$ which completes the proof.

Proof of Theorem 1.4 By Theorem 1.1, we have

$$
\begin{aligned}
m^{-}\left(\Lambda_{s} \oplus \Lambda_{e}\right)-m^{-}\left(\Lambda_{s} \oplus \Lambda_{D}^{n}\right) & =\left(m^{-}\left(\Lambda_{s} \oplus \Lambda_{e}\right)-m^{-}\left(\Lambda_{D}\right)\right)-\left(m^{-}\left(\Lambda_{s} \oplus \Lambda_{D}^{n}\right)-m^{-}\left(\Lambda_{D}\right)\right) \\
& \left.\left.=i\left(G r(\gamma(T)), \Lambda_{s} \oplus \Lambda_{e}, \Lambda_{D}\right)\right)-i\left(G r(\gamma(T)), \Lambda_{s} \oplus \Lambda_{D}^{n}, \Lambda_{D}\right)\right) \\
& \left.=i(\gamma(T)) \Lambda_{s}, \Lambda_{e}, \Lambda_{D}^{n}\right),
\end{aligned}
$$

where the last equality is from 2.15), 2.18). 
Then (3.22) derives that

$$
\left.m^{-}\left(\Lambda_{s} \oplus \Lambda_{e}\right)=m^{-}\left(\Lambda_{s} \oplus \Lambda_{D}^{n}\right)+i(\gamma(T)) \Lambda_{s}, \Lambda_{e}, \Lambda_{D}^{n}\right)
$$

The rest work is to compute $m^{-}\left(\Lambda_{s} \oplus \Lambda_{D}^{n}\right)$ by using Maslov index. In fact, for every $t \in[0, T]$ and $z=$ $\gamma(t)^{-1}(y, 0)^{T} \in \gamma(t) \Lambda_{s} \cap \Lambda_{D}^{n}$, by some direct computations the crossing form is given by

$$
\left\langle-\gamma(t)^{T} J \dot{\gamma}(t) z, z\right\rangle=\left\langle\mathcal{B}(t)\left[\begin{array}{l}
y \\
0
\end{array}\right],\left[\begin{array}{l}
y \\
0
\end{array}\right]\right\rangle=\left\langle P^{-1}(t) y, y\right\rangle>0
$$

where $\mathcal{B}(t)$ is defined in (1.6). Moreover, one can easily check that $\operatorname{Gr}(\gamma(0)) \cap\left(\Lambda_{s} \oplus \Lambda_{D}^{n}\right)=\operatorname{Gr}\left(I_{2 n}\right) \cap\left(\Lambda_{s} \oplus\right.$ $\left.\Lambda_{D}^{n}\right)=\operatorname{dim}\left(\Lambda_{s} \cap \Lambda_{D}^{n}\right)$ and $\operatorname{Gr}(\gamma(t)) \cap\left(\Lambda_{s} \oplus \Lambda_{D}^{n}\right)=\operatorname{dim}\left(\gamma(t) \Lambda_{s} \cap \Lambda_{D}^{n}\right)$ for every $t \in(0, T)$. Then by the formula (2.2) we have

$$
\mu\left(\Lambda_{s} \oplus \Lambda_{D}^{n}, G r(\gamma(t))\right)=\sum_{0<t<T} \operatorname{dim}\left(\gamma(t) \Lambda_{s} \cap \Lambda_{D}^{n}\right)+\operatorname{dim}\left(\Lambda_{s} \cap \Lambda_{D}^{n}\right) .
$$

By Corollary 1.6, we have

$$
m^{-}\left(\Lambda_{s} \oplus \Lambda_{D}^{n}\right)=\mu\left(\Lambda_{s} \oplus \Lambda_{D}^{n}, G r(\gamma(t))\right)-n+i\left(G r\left(I_{2 n}\right), \Lambda_{s} \oplus \Lambda_{D}^{n}, \Lambda_{D}\right)
$$

Recall that $i\left(\operatorname{Gr}\left(I_{2 n}\right), \Lambda_{s} \oplus \Lambda_{D}^{n}, \Lambda_{D}\right)=n-\operatorname{dim}\left(\Lambda_{s} \cap \Lambda_{D}^{n}\right)$, together with (3.24) then (3.25) is converted into

$$
m^{-}\left(\Lambda_{s} \oplus \Lambda_{D}^{n}\right)=\sum_{0<t<T} \operatorname{dim}\left(\gamma(t) \Lambda_{s} \cap \Lambda_{D}^{n}\right)
$$

By (3.23) and (3.25) we have

$$
\left.m^{-}\left(\Lambda_{s} \oplus \Lambda_{e}\right)=\sum_{0<t<T} \operatorname{dim}\left(\gamma(t) \Lambda_{s} \cap \Lambda_{D}^{n}\right)+i(\gamma(T)) \Lambda_{s}, \Lambda_{e}, \Lambda_{D}^{n}\right) .
$$

This conclude the proof.

Example 3.8 (Index Theorem of [14]). Let $(M, g)$ be a complete Riemannian manifold of dimension d and $c:[0, T] \rightarrow M$ be a geodesic. Assume $P$ and $Q$ are two submanifolds of $M$ such that

$$
c(0) \in P, \quad \dot{c}(0) \perp P_{c(0)}, \quad c(T) \in Q, \quad \dot{c}(T) \perp Q_{c(T)},
$$

where the dimension of $Q_{c(T)}$ is $r$ such that $0 \leq r<d$.

Let $\left\{e_{1}(0), \ldots, e_{d-1}(0)\right\}$ be an orthonormal basis of normal bundle $N_{c(0)} M$ at $c(0)$, then by parallel trivialization along $c$, we get an orthonormal basis $\left\{e_{1}(t), \ldots, e_{d-1}(t)\right\}$ of $N_{c(t)} M$. Let $\hat{R}=\hat{R}(\dot{c}, X) \dot{c}$ be the curvature tensor of the Levi-Civita connection. For every Jacobi field along $c$ given by $X(t)=\sum_{i=1}^{d-1} u_{i}(t) e_{i}(t)$, the Jacobi equation

$$
-X^{\prime \prime}+\hat{R} X=0
$$

will be reduced to

$$
-u^{\prime \prime}(t)+R(t) u(t)=0,
$$

where $u(t)=\left(u_{1}(t), \ldots, u_{d-1}(t)\right)^{T}$ and $R(t)=\left[R_{i, j}(t)\right]_{i, j=1}^{d-1}$ with $R_{i, j}(t)=\left\langle\hat{R}\left(\dot{c}(t), e_{i}(t)\right) \dot{c}(t), e_{j}(t)\right\rangle$. 
Let $\hat{S}(t)$ be the second fundamental form of $P$ at $c(t)$ with respect to $\dot{c}(t)$. Denote $S(t)=\left[S_{i, j}(t)\right]_{i, j=1}^{d-1}$ with $S_{i, j}(t)=\left\langle\hat{S}(t) e_{i}(t), e_{j}(t)\right\rangle$. Then $R(t)$ and $S(t)$ are both symmetric. The index form

$$
\hat{I}(X, Y)=\int_{0}^{T}\left\langle\hat{R} X-X^{\prime \prime}, Y\right\rangle d t+\left.\left\langle X^{\prime}-\hat{S} X, Y\right\rangle\right|_{0} ^{T}
$$

is converted into

$$
I(u, v)=\int_{0}^{T}\left\langle R u-u^{\prime \prime}, v\right\rangle d t+\left.\left\langle u^{\prime}-S u, v\right\rangle\right|_{0} ^{T}
$$

Since $\hat{I}$ is defined on $H=\left\{V(t)=\sum_{i=1}^{d-1} u_{i}(t) e_{i}(t), V(0) \in P_{c(0)}, V(T) \in Q_{c(T)}\right\}$, namely, the linear space of continuous piecewise $C^{\infty}$ vector fields along $\gamma$ which are orthogonal to $\gamma$ and whose initial and final vectors are in $P_{c(0)}$ and $Q_{c(T)}$ respectively, then I is defined on $\mathcal{H}=\left\{u \in W^{1,2}\left([0, T], \mathbf{R}^{d-1}\right), u(0) \in V_{0}, u(T) \in V_{T}\right\}$, where $V_{0}, V_{T} \subset \mathbf{R}^{d-1}$ can be considered as the tangent space of $P, Q$ at $c(0), c(T)$ separately. Note that the index form $\hat{I}$ is a little different from the original one in $[14]$ since we have assumed that $X^{\prime}$ is continuous, this is not an essential problem.

A Jacobi field $X$ is called $P$-Jacobi field if it is orthogonal to $c$ such that $X(0) \in P_{c(0)}$ and $X^{\prime}(0)-S_{0} X(0) \perp$ $P_{c(0)}$. If $X(t)=\sum_{i=1}^{d-1} u_{i}(t) e_{i}(t)$ is a P-Jacobi field then by direct computations we have $u(t)$ is a solution of system (3.28) such that $u(0) \in V_{0}$ and $u^{\prime}(0)-S(0) u(0) \in V_{0}^{\perp}$. A P-focal point is a point $c\left(t_{0}\right), t_{0} \in(0, T]$ such that there exists a nonzero $P$-Jacobi field which vanishes at $t_{0}$.

Let $y(t)=u^{\prime}(t)$ and $z(t)=(y(t), x(t))^{T}$, then system (3.28) is converted into Hamiltonian system (1.5) with $\mathcal{B}(t)=\left[\begin{array}{cc}I_{n} & 0 \\ 0 & -R\end{array}\right]$ and the boundary condition is given by

$$
z(0) \in \Lambda_{s}:=\left\{y(0)-S(0) x(0) \in J_{d-1} V_{0}^{\perp}\right\}, z(T) \in \Lambda_{e}:=\left\{y(T)-S(T) x(T) \in J_{d-1} V_{T}^{\perp}\right\} .
$$

Let $\gamma(t)$ be the fundamental solution. In fact, after parallel trivialization, a P-focal point is equivalent to $\Lambda_{s}$ conjugate point which is defined above Theorem 1.4 then by Theorem 1.4 we have

$$
m^{-}\left(\Lambda_{s} \oplus \Lambda_{e}\right)=\sum_{0<t_{0}<T} \operatorname{dim}\left(\gamma\left(t_{0}\right) \Lambda_{s} \cap \Lambda_{D}^{n}\right)+i\left(\gamma(T) \Lambda_{s}, \Lambda_{e}, \Lambda_{D}^{n}\right) .
$$

Note that $\operatorname{dim}\left(\gamma\left(t_{0}\right) \Lambda_{s} \cap \Lambda_{D}^{n}\right)$ is just the multiplicity of P-focal point $c\left(t_{0}\right)$ and $i\left(\gamma(T) \Lambda_{s}, \Lambda_{e}, \Lambda_{D}^{n}\right)$ is just the difference $m^{-}\left(\Lambda_{s} \oplus \Lambda_{e}\right)-m^{-}\left(\Lambda_{s} \oplus \Lambda_{D}^{n}\right)$ which is equal to $i(A)$ in [14] under the non-degenerate assumption. We conclude this example.

\section{The stability of brake orbits}

Recall that a brake orbit $x(t)$ of system (1.3) satisfies that

$$
\dot{x}(0)=\dot{x}(T / 2)=0, \quad x(T / 2+t)=x(T / 2-t), \quad x(T+t)=x(t), \quad \forall t \in \mathbf{R} .
$$

Let $\mathcal{N}=\left[\begin{array}{cc}-I_{n} & 0 \\ 0 & I_{n}\end{array}\right]$, then the corresponding brake orbit $z(t)$ of system (1.5) satisfies that

$$
z(-t)=\mathcal{N} z(t), \quad z(t+T)=z(t), \quad \forall t \in \mathbf{R} .
$$

It's easy to check that

$$
(\mathcal{N} \gamma(T))^{2}=I_{2 n}, \quad \gamma(T)=\mathcal{N} \gamma\left(\frac{T}{2}\right)^{-1} \mathcal{N} \gamma\left(\frac{T}{2}\right) .
$$

Before to give another corollary of Theorem 1.1 and the application to brake orbits, we need an important but simple lemma. 
Lemma 4.1. For a given linear subspace $\mathcal{W} \subset \mathbf{R}^{n}$, let $\mathfrak{Q}$ be a quadratic form defined on $\mathcal{W}$ and $\mathcal{X} \subset \mathcal{W}$ be any subspace such that $\mathfrak{Q}(x, y)=0$ for every $x, y \in X$. Then we have

$$
\operatorname{dim} \mathcal{X} \leq m^{+}(\mathfrak{Q})+\operatorname{dim} \operatorname{ker} \mathfrak{Q} .
$$

Particularly, if we have the splitting $\operatorname{ker} \mathfrak{Q}=\operatorname{ker}_{1} \mathfrak{Q} \oplus \operatorname{ker}_{2} \mathfrak{Q}$ and $\mathcal{X} \cap \operatorname{ker}_{1} \mathfrak{Q}=\{0\}$, then for $\mathcal{X}$ as above there holds

$$
\operatorname{dim} \mathcal{X} \leq m^{+}(\mathfrak{Q})+\operatorname{dim} \operatorname{ker}_{2} \mathfrak{Q} .
$$

Proof. Associated to $\mathfrak{Q}$, there exists a symmetric matrix $\mathcal{A}$ such that $\mathfrak{Q}(x, y)=\langle\mathcal{A} x, y\rangle$, then we have the splitting

$$
\mathcal{W}=\mathcal{W}^{+} \oplus \mathcal{W}^{0} \oplus \mathcal{W}^{-},
$$

where $\mathcal{W}^{*}, *=+, 0,-$ denote the corresponding positive, zero and negative eigenspaces of $\mathcal{A}$. $\operatorname{If} \operatorname{dim} \mathcal{X}>$ $m^{+}(\mathfrak{Q})+\operatorname{dim} \operatorname{ker} \mathfrak{Q}$, then $\mathcal{X} \cap \mathcal{W}^{-} \neq\{0\}$ which is a contradiction to $\mathfrak{Q}(x, y)=0$ for every $x, y \in \mathcal{X}$.

For the second statement, if (4.5) is false, then $X \cap\left(\operatorname{ker}_{1} \mathfrak{Q} \oplus \mathcal{W}^{-}\right) \neq\{0\}$. There must exist a nonzero $x=$ $x_{0}^{1}+x^{-} \in \mathcal{X}$ with $x_{0}^{1} \in \operatorname{ker}_{1} \mathfrak{Q}, x^{-} \in \mathcal{W}$ such that $\mathfrak{Q}(x, x)=\mathfrak{Q}\left(x_{0}^{1}, x_{0}^{1}\right)+\mathfrak{Q}\left(x^{-}, x^{-}\right)=0$. It derives $\mathfrak{Q}\left(x^{-}, x^{-}\right)=0$ and consequently $x^{-}=0$. Hence $x=x_{0}^{1} \in \operatorname{ker}_{1} \mathfrak{Q} \cap \mathcal{X}$ which is to contradiction to $\mathcal{X} \cap \operatorname{ker}_{1} \mathfrak{Q}=\{0\}$. We complete the proof.

Now we can prove another corollary of Theorem 1.1 as following:

Corollary 4.2. Let $\gamma(T)$ be the monodromy matrix of system (1.5) and $\mathbf{U}$ be the unit circle of complex plane, then

$$
\operatorname{dim} \operatorname{ker}\left(\gamma(T)-\lambda I_{2 n}\right) \leq m^{-}\left(\Lambda_{N}\right)+m^{0}\left(\Lambda_{N}\right)-m^{-}\left(\Lambda_{D}\right) .
$$

Proof. Let $\gamma(T)=\left[\begin{array}{ll}D_{1} & D_{2} \\ D_{3} & D_{4}\end{array}\right]$, please recall that the frame of $\operatorname{Gr}(\gamma)$ under the symplectic form $J_{2 n}$ is given by $\left[\begin{array}{cc}-I & 0 \\ D_{1} & D_{2} \\ 0 & I \\ D_{3} & D_{4}\end{array}\right]$. For every $\lambda \in \sigma(\gamma(T))$ with corresponding eigenvector $u_{\lambda}=(x, y)^{T}$, namely, $\gamma(T) u_{\lambda}=\lambda u_{\lambda}$, let

$$
z_{\lambda}=\left(-x, D_{1} x+D_{2} y, y, D_{3} x+D_{4} y\right)^{T} \in G r(\gamma(T)) \cap\left(\Lambda_{N}+\Lambda_{D}\right) .
$$

By the definition of $\mathfrak{Q}\left(\operatorname{Gr}(\gamma(T)), \Lambda_{N} ; \Lambda_{D}\right)$ and direct computations we have

$$
\mathfrak{Q}\left(z_{\lambda}, z_{\lambda}\right)=\langle x, y\rangle-\left\langle D_{1} x+D_{2} y, D_{3} x+D_{4} y\right\rangle .
$$

For every $\lambda \in \sigma(\gamma(T)) \cap \mathbf{U}$ and every $(x, y)^{T} \in \operatorname{ker}\left(\gamma(T)-\lambda I_{2 n}\right)$ which derives $D_{1} x+D_{2} y=\lambda x, D_{3} x+D_{4} y=\lambda y$, then by (4.7) we have $\mathfrak{Q}\left(z_{\lambda}, z_{\lambda}\right)=0$. By Lemma 4.1 we get

$$
\operatorname{dim} \operatorname{ker}\left(\gamma(T)-\lambda I_{2 n}\right) \leq m^{+}(\mathfrak{Q})+\operatorname{dim} \operatorname{ker} \mathfrak{Q} .
$$

But note that

$$
\operatorname{ker} \mathfrak{Q}=\left(G r(\gamma(T)) \cap \Lambda_{D}\right) \oplus\left(G r(\gamma(T)) \cap \Lambda_{N}\right),
$$

then

$$
\operatorname{dim} \operatorname{ker}\left(\gamma(T)-\lambda I_{2 n}\right) \leq i\left(G r(\gamma(T)), \Lambda_{N}, \Lambda_{D}\right)+\operatorname{dim} \operatorname{Gr}(\gamma(T)) \cap \Lambda_{N} .
$$

From the fact that $m^{0}\left(\Lambda_{N}\right)=\operatorname{dim} G r(\gamma(T)) \cap \Lambda_{N}$ and combine with Theorem 1.1 and (4.8), we complete the proof. 
Now we will go on the application to a brake orbit. We still denote $\gamma(T)=\left[\begin{array}{ll}D_{1} & D_{2} \\ D_{3} & D_{4}\end{array}\right]$ and $\gamma(T / 2)=$ $\left[\begin{array}{ll}E_{1} & E_{2} \\ E_{3} & E_{4}\end{array}\right]$. Since $\gamma(T / 2) \in \operatorname{Sp}(2 n)$, then

$$
E_{1}^{T} E_{3}=E_{3}^{T} E_{1}, \quad E_{2}^{T} E_{4}=E_{4}^{T} E_{2}, \quad E_{4}^{T} E_{1}-E_{2}^{T} E_{3}=I_{n} .
$$

From $\gamma(T)=\mathcal{N} \gamma^{-1}(T / 2) \mathcal{N} \gamma(T / 2) \in \operatorname{Sp}(2 n)$, direct computations show that

$$
D_{1}=E_{4}^{T} E_{1}+E_{2}^{T} E_{3}, \quad D_{2}=E_{4}^{T} E_{2}+E_{2}^{T} E_{4}, \quad D_{3}=E_{3}^{T} E_{1}+E_{1}^{T} E_{3}, \quad D_{4}=E_{3}^{T} E_{2}+E_{1}^{T} E_{4},
$$

and we have

$$
D_{4}=D_{1}^{T}, \quad D_{2}=D_{2}^{T}, \quad D_{3}=D_{3}^{T}, \quad D_{1}^{T} D_{3}=D_{3} D_{1}, \quad D_{2} D_{1}^{T}=D_{1} D_{2}, \quad D_{1}^{2}-D_{2} D_{3}=I_{n} .
$$

We start from a simple lemma.

Lemma 4.3. For $\lambda_{1}, \lambda_{2} \in \sigma(\gamma(T))$ with corresponding eigenvectors $u_{1}=\left(x_{1}, y_{1}\right)^{T}, u_{2}=\left(x_{2}, y_{2}\right)^{T}$, then there must hold at least one of the three statements: (1) $\lambda_{1}=\bar{\lambda}_{2}, \quad$ (2) $\lambda_{1} \bar{\lambda}_{2}=1 \quad$ (3) $\left\langle x_{1}, y_{2}\right\rangle=0$.

Proof. For every $\lambda \in \sigma(\gamma(T))$ with corresponding eigenvector $u_{\lambda}=(x, y)^{T}$, namely, $\gamma(T) u_{\lambda}=\lambda u_{\lambda}$, by (4.3) we have $\gamma(T) \mathcal{N} u_{\lambda}=\mathcal{N} \gamma(T)^{-1} u_{\lambda}=\lambda^{-1} \mathcal{N} u_{\lambda}$, then $\mathcal{N} u_{\lambda}=(-x, y)^{T}$ is an eigenvector related to $\lambda^{-1} \in \sigma(\gamma(T))$. By the expression of $\gamma(T)$ and some direct calculations, there hold

$$
D_{1} x=\frac{\lambda+\lambda^{-1}}{2} x, D_{2} y=\frac{\lambda-\lambda^{-1}}{2} y, D_{3} x=\frac{\lambda-\lambda^{-1}}{2} y, D_{4} y=\frac{\lambda+\lambda^{-1}}{2} y .
$$

For $\lambda_{1}, \lambda_{2} \in \sigma(\gamma(T))$ with corresponding eigenvectors $u_{1}=\left(x_{1}, y_{1}\right)^{T}, u_{2}=\left(x_{2}, y_{2}\right)^{T}$, then by (4.10) and (4.11) we have

$$
\left\langle\frac{\lambda_{1}+\lambda_{1}^{-1}}{2} x_{1}, y_{2}\right\rangle=\left\langle D_{1} x_{1}, y_{2}\right\rangle=\left\langle x_{1}, D_{1}^{*} y_{2}\right\rangle=\left\langle x_{1}, D_{4} y_{2}\right\rangle=\left\langle x_{1}, \frac{\lambda_{2}+\lambda_{2}^{-1}}{2} y_{2}\right\rangle
$$

which derives

$$
\left(\frac{\lambda_{1}+\lambda_{1}^{-1}}{2}-\frac{\bar{\lambda}_{2}+\bar{\lambda}_{2}^{-1}}{2}\right)\left\langle x_{1}, y_{2}\right\rangle=0
$$

In fact, $\frac{\lambda_{1}+\lambda_{1}^{-1}}{2}-\frac{\bar{\lambda}_{2}+\bar{\lambda}_{2}^{-1}}{2}=0$ is equivalent to $\lambda_{1}=\bar{\lambda}_{2}$ or $\lambda_{1} \bar{\lambda}_{2}=1$. By (4.12) we complete the proof.

Corollary 4.4. For $i=1,2, \lambda_{i} \in \sigma(\gamma(T))$ with eigenvector $u_{i}$, let $z_{i}=\left(-x_{i}, D_{1} x_{i}+D_{2} y_{i}, y_{i}, D_{3} x_{i}+D_{4} y_{i}\right)^{T}$, then as long as $\lambda_{1} \neq \bar{\lambda}_{2}$ or $\lambda_{1}=\lambda_{2}= \pm 1$, we have $z_{1}$ and $z_{2}$ are $\mathfrak{Q}$-orthogonal.

Proof. Consider the form $\mathfrak{Q}\left(\operatorname{Gr}(\gamma(T)), \Lambda_{N} ; \Lambda_{D}\right)$ defined on $\operatorname{Gr}(\gamma(T)) \cap\left(\Lambda_{N}+\Lambda_{D}\right)$ and let $\lambda_{i}, u_{i}, i=1,2$ be as above, then by (4.7) and direct computations we have

$$
\mathfrak{Q}\left(z_{1}, z_{2}\right)=\left(1-\lambda_{1} \bar{\lambda}_{2}\right)\left\langle x_{1}, y_{2}\right\rangle
$$

Obviously, if $1=\lambda_{1} \bar{\lambda}_{2}$, then $z_{1}$ and $z_{2}$ are Q-orthogonal. If $1 \neq \lambda_{1} \bar{\lambda}_{2}$ and $\lambda_{1} \neq \bar{\lambda}_{2}$, by Lemma 4.3 , we still have $z_{1}$ and $z_{2}$ are $\mathfrak{Q}$-orthogonal.

Now we give the proof of Theorem 1.8 . 
Proof of Theorem 1.8. For every $\lambda \in \sigma(\gamma(T))$ with corresponding eigenvector $u_{\lambda}=(x, y)^{T}$, we denote $z=\left(-x, D_{1} x+D_{2} y, y, D_{3} x+D_{4} y\right)^{T}$. In fact, it's apparent that $\lambda_{1} \neq \bar{\lambda}_{2}$ for any $\lambda_{1}, \lambda_{2} \in \sigma(\gamma(T)) \cap \mathbf{C}^{\perp} \cup\{ \pm 1\}$, then by Corollary 4.4, we have $z_{1}, z_{2}$ are $\mathfrak{Q}$-orthogonal. We get (1.22) from Lemma 4.1

To prove (1.23), recall that $\operatorname{ker} \mathfrak{Q}\left(\operatorname{Gr}(\gamma(T)), \Lambda_{N} ; \Lambda_{D}\right)=\operatorname{ker}_{1} \mathfrak{Q} \oplus \operatorname{ker}_{2} \mathfrak{Q}$ where $\operatorname{ker}_{1} \mathfrak{Q}=\operatorname{Gr}(\gamma(T)) \cap \Lambda_{N}$ and $\operatorname{ker}_{2} \mathfrak{Q}=\operatorname{Gr}(\gamma(T)) \cap \Lambda_{D}$. For every $\lambda \in \sigma(\gamma(T)) \cap \mathbf{C}^{\perp}$, if $z=\left(-x, D_{1} x+D_{2} y, y, D_{3} x+D_{4} y\right)^{T} \in \Lambda_{N}$, then $x=0$. Together with (4.11) which derives $D_{3} x=\frac{\lambda-\lambda^{-1}}{2} y=0$, then $y=0$ since $\frac{\lambda-\lambda^{-1}}{2} \neq 0$. So $z \notin \Lambda_{N}$. Note that for $\lambda_{i}, \lambda_{j} \in \sigma(\gamma(T)) \cap \mathbf{C}^{\perp}$, there must hold $\frac{\lambda_{i}+\lambda_{i}^{-1}}{2} \neq \frac{\lambda_{j}+\lambda_{j}^{-1}}{2}$ if $\lambda_{i} \neq \lambda_{j}$. By 4.11) we know $D_{1} x_{i}=\frac{\lambda_{i}+\lambda_{i}^{-1}}{2} x_{i}$ which deduces that all $x_{i}$ are linear independent for different $\lambda_{i}$. If there is a linear combination $\sum_{i} a_{i} z_{i} \in \Lambda_{N}$, then we have $\sum_{i} a_{i} x_{i}=0$ and consequently every $a_{i}=0$. Then every $z=\sum_{i} a_{i} z_{i}$ is not in $\Lambda_{N}$ which means $\operatorname{ker} \mathfrak{Q} \cap \operatorname{Gr}(\gamma(T)) \cap \Lambda_{N}=\{0\}$. Let $\mathcal{X}$ be the space of all $z=\left(-x, D_{1} x+D_{2} y, y, D_{3} x+D_{4} y\right)^{T}$ such that $(x, y)^{T}$ is an eigenvector of some $\lambda \in \sigma(\gamma(T)) \cap \mathbf{C}^{\perp}$. Then $\left.\mathfrak{Q}\right|_{\mathcal{X}}=0$ and $\mathcal{X} \cap \operatorname{ker}_{1} \mathfrak{Q}=\{0\}$. By (4.5) there hold

$$
\operatorname{dim} \bigoplus_{\lambda \in \sigma(\gamma(T)) \cap \mathbf{C}^{\perp}} \operatorname{ker}\left(\gamma(T)-\lambda I_{2 n}\right) \leq m^{+}(\mathfrak{Q})+\operatorname{dim}\left(\operatorname{Gr}(\gamma(T)) \cap \Lambda_{D}\right) .
$$

Recall the formula (2.8) and Theorem 1.1, the righthand side of (4.14) is exactly $m^{-}\left(\Lambda_{N}\right)-m^{-}\left(\Lambda_{D}\right)$. We complete the proof.

Now we can present the proof of Theorem 1.9

Proof of Theorem 1.9 Let $m^{-}\left([a, b] ; \Lambda_{0}\right)$ be the Morse index on time interval $[a, b]$ for the boundary condition $\Lambda_{0}$. Denote $V_{ \pm}(\mathcal{N})$ the eigenspace corresponding to the eigenvalue \pm 1 of $\mathcal{N}$. By simple computations we have $V_{+}(\mathcal{N})=\Lambda_{N}^{n}$ and $V_{-}(\mathcal{N})=\Lambda_{D}^{n}$. Then by [12, Theorem 2] we have

$$
\begin{aligned}
& k=m^{-}\left(x, \Lambda_{P}\right)=m^{-}\left([0, T / 2] ; \Lambda_{N}\right)+m^{-}\left([0, T / 2] ; \Lambda_{D}\right) \\
& m^{-}\left([0, T] ; \Lambda_{N}\right)=m^{-}\left([0, T / 2] ; \Lambda_{N}\right)+m^{-}\left([0, T / 2] ; \Lambda_{N}^{n} \oplus \Lambda_{D}^{n}\right) \\
& m^{-}\left([0, T] ; \Lambda_{D}\right)=m^{-}\left([0, T / 2] ; \Lambda_{D}\right)+m^{-}\left([0, T / 2] ; \Lambda_{D}^{n} \oplus \Lambda_{N}^{n}\right)
\end{aligned}
$$

which obviously derives

$$
m^{-}\left(\Lambda_{N}\right)-m^{-}\left(\Lambda_{D}\right) \leq 2 k
$$

By (1.23) we complete the proof.

As a corollary, we give a new proof for Ureña's [22, Theorem 1.1] interesting result.

Theorem 4.5. For a minimizer brake orbit all eigenvalues of monodromy matrix $\gamma(T)$ are real and positive.

Proof. Recently, in [22, Theorem 1.1] the author proves an great result that for a minimizer brake orbit all eigenvalues of monodromy matrix $\gamma(T)$ are real and positive. In fact, this result can be easily proved by Theorem 1.1 and Theorem 1.9. Precisely, since the brake orbit is a minimizer, then the Morse index $k=0$ in Theorem 1.9 which means all eigenvalues of $\gamma(T)$ are real. Moreover, $m^{-}\left(\Lambda_{N}\right)=m^{-}\left(\Lambda_{D}\right)=0$. The rest work is only to prove all $\lambda \in \sigma(\gamma(T))$ are positive. By theorem 1.1 and (2.8), we have

$$
\mathfrak{Q}\left(G r(\gamma(T)), \Lambda_{N} ; \Lambda_{D}\right) \leq 0, \quad G r(\gamma(T)) \cap \Lambda_{D}=\{0\} .
$$

In fact, $\operatorname{Gr}(\gamma(T)) \cap \Lambda_{D}=\{0\}$ can derive $D_{3}$ is invertible. By 44.7) and $\mathfrak{Q}\left(\operatorname{Gr}(\gamma(T)), \Lambda_{N} ; \Lambda_{D}\right) \leq 0$ we have $D_{3} D_{1} \geq 0$. By (4.3) and (4.9), we have $D_{3}=2 E_{3}^{T} E_{1}$. By (4.15), we have $m^{-}\left([0, T / 2] ; \Lambda_{N}\right)=$ 
$m^{-}\left([0, T / 2] ; \Lambda_{D}\right)=0$. By Theorem 1.1 again, we have $E_{3}^{T} E_{1} \geq 0$. Consequently, $D_{3} \geq 0$. Recall that $D_{3}$ is invertible, then $D_{3}>0$. Since $D_{3}^{-1 / 2}\left(D_{3} D_{1}\right) D_{3}^{-1 / 2}$ is similar to $D_{1}$, then $D_{1} \geq 0$. By (4.11), there hold for every $\lambda \in \sigma(\gamma(T))$ is equivalent to $\frac{\lambda+\lambda^{-1}}{2} \in \sigma\left(D_{1}\right)$, then there must hold all $\lambda \in \sigma(\gamma(T))$ are positive.

Acknowledgements. The authors sincerely thank Professor Alessandro Portaluri for the discussions of index theory and the stability problem of brake orbits.

\section{References}

[1] Abbondandolo, A., On the Morse index of Lagrangian systems. Nonlinear Anal., 53, (2003), 551-566.

[2] Ambrose W., The Index Theorem in Riemannian Geometry., Annals of Mathematics, 73, (1961), no.1, 49-86.

[3] Arnold, V. I., Characteristic class entering in quantization conditions, Funkts. Anal, Priloch., 1, (1967), no.1, 1-13.

[4] Ballmann, W., Thobergsson, G., Ziller, W., Closed geodesics on positively curved manifolds. Ann. of Math., 116(1982), no. 2, 213-247.

[5] Bolton J., The Morse index theorem in the case of two variable end-points., Journal of Differential Geometry, 12(1977), 567-581.

[6] Bolotin, S.V., Treschev, D.V., Hill’s formula. Russ. Math. Surv. 65(2),191-257(2010).

[7] Cappell, S.E., Lee, R., Miller, E.Y., On the maslov index. Comm.Pure Appl. Math., 47(1994), no.2, 121-186.

[8] Courant, R., Hilbert, D.: Methods of Mathematical Physics, vol. I. Interscience Publishers, Inc., New York (1953).

[9] Duistermaat, J.J., On the Morse index in variational calculus. Advances in Mathematics, 21(1976), no.2, 173-195.

[10] Edwards, H. M., A Generalized Sturm Theorem., Annals of Mathematics, 80(1964), no.1, 22-57.

[11] Howard P., Sukhtayev A., The Maslov and Morse indices for Schrodinger operators on [0,1]. Journal of Differential Equations, 260 (2016), no. 5, 4499-4549.

[12] Hu, X., Portaluri, A., Yang, R., A dihedral Bott-type iteration formula and stability of symmetric periodic orbit. Preprint available on https://arxiv.org/pdf/1705.09173.pdf.

[13] Hu, X., Sun, S., Index and stability of symmetric periodic orbits in Hamiltonian systems with its application to figure-eight orbit. Commun. Math. Phys. 290 (2009), no. 2, 737-777.

[14] Kalish D., The Morse index theorem where the ends are submanifolds., Transactions of the American Mathematical Society, 308 (1988), no. 1, 341-348.

[15] Long, Y., Index Theory for Symplectic Paths with Applications, Progress in Math. 207, Birkhäuser. Basel. 2002. 
[16] Long, Y., An, T., Indexing domains of instability for Hamiltonian systems., NoDEA Nonlinear Differential Equations Appl, 5,(1998), no.4, 461-478.

[17] Long, Y., Zhu, C., Maslov-type index theory for symplectic paths and spectral flow (II)., Chin.Ann.of Math, 21, (2000), no.1, 89-108.

[18] Müller-Pfeiffer, E., Spectral Theory of Ordinary Differential Operators. E. Horwood, 1981.

[19] Morse, M., The calculus of variation in the large. Colloquium Publication, Volume 18. Providence, RI:AMS, 1934.

[20] Offin, D., A spectral theorem for reversible second order equations with periodic coefficients. Differential Integral Equations 5 (1992), no. 3, 615-629.

[21] Robbin, J., Salamon, D., The Maslov index for paths. Topology, 32(1993), no.4, 827-844.

[22] Ureña, A.J., The spectrum of reversible minimizers. Preprint 2017.

[23] Weber, J., Perturbed closed geodesics are periodic orbits: Index and transversality. Mathematische Zeitschrift, 241(2002), no.1, 45-81.

[24] Wu, L., The Calculation of Maslov-type index and Hörmonder index in weak symplectic Banach space. Ph.D Thesis, Nankai University, 2016.

[25] Zhou, Y., Wu, L., Zhu, C., The Hörmander index in the finite dimensional case, Front. Math. China, to appear.

[26] Zhu, C., A generalized Morse index theorem. Analysis, geometry and topology of elliptic operators, World Sci. Publ., Hackensack, NJ, 2006, 493-540.

[27] Zhu, C., Long, Y., Maslov-type index theory for symplectic paths and spectral flow (I)., Chin.Ann.of Math, 20B(1999), no.4, 413-424 . 